\title{
Domino reactions of cyclic enaminones leading to selective synthesis of pentacyclic indoles and its functionalization
}

\author{
Wei Fan', Yan-Rong Li ${ }^{\mathrm{a}}$, Qun $\mathrm{Li}^{\mathrm{a}}$, Bo Jiang ${ }^{\mathrm{b}, \mathrm{c},}$, Guigen $\mathrm{Li}^{\mathrm{a}}$, , ** \\ ${ }^{a}$ Institute of Chemistry \& BioMedical Sciences, Nanjing University, Nanjing 210093, P. R. China; ${ }^{b}$ Department of Chemistry and Biochemistry, Texas Tech \\ University, Lubbock, Texas 79409-1061, United States; 'School of Chemistry and Chemical Engineering, Jiangsu Normal University, Xuzhou 221116, Jiangsu, \\ P.R. China; Corresponding author.e-mail: jiangchem@jsnu.edu.cn (B.Jiang), guigen.li@ttu.edu (G. Li)
}

\section{ARTICLE INFO}

Article history:

Received

Received in revised form

Accepted

Available online

\section{Keywords:}

Multicomponent domino reactions (MDRs)

Fused indoles

Allylic hydroxylation

Esterification

Group-Assisted-Purification (GAP) chemistry

\section{ABSTRACT}

A new multicomponent domino reaction of cyclic enaminones with acenaphthylene-1,2-dione in a anhydride solvent has been established, providing selective protocol to pentacyclic indoles with different substituted patterns (up to 50 examples). Both substitutions on the cyclic enaminone ring and reaction temperatures showed obvious impact on the reaction pathways. For instance, selective allylic hydroxylation and allylic esterification of in-situ generated indoles depend on reaction temperatures. With special substituents, the reactions underwent dehydrogenation process to give access to different substituted fused indoles with good to excellent yields. These reactions feature mild conditions, convenient one-pot operation and short reaction times. The purification of products only needs to be washed by $95 \%$ ethanol without traditional chromatography and recrystallization, which characterizes group-assisted purification (GAP) chemistry.

2016 Elsevier Ltd. All rights reserved.

\section{Introduction}

The assembly of complex polycyclic frameworks of chemical and biological interest has emerged as an active theme in modern organic chemistry. ${ }^{1,2}$ Among these systems, the structurally diverse polycyclic indoles commonly exists in natural alkaloids ${ }^{3}$ and often referred to as "privileged medicinal scaffolds" in drug discovery because of their special chemical and biological activities. ${ }^{4}$ Compounds containing this polycyclic indole moiety have been served as $3 \alpha$-hydroxysteroid dehydrogenase inhibitors, ${ }^{5}$ DNA intercalators, topoisomerase II inhibitors, ${ }^{6}$ lipid peroxidation inhibitors, ${ }^{7}$ and potassium channel openers. ${ }^{8}$ Accordingly, diverse approaches to polycyclic indoles have been established. ${ }^{9}$ However, most of these methods suffered from drawbacks including the use of transition-metal catalysts, ${ }^{10}$ limited availability of starting materials, multistep sequences, incompatibility with various functional groups and harsh reaction conditions. Therefore, the development of reaction sequences that are short, convenient to carry out, and efficient toward complex and diverse polycyclic indoles from simple substrates continues to be of great interest in the scientific community.

Multicomponent domino reactions (MDRs) for total synthesis of natural products or natural-like structures are considered to be one of the powerful tools in the modern drug discovery and development process, enabling rapid access to structural variation and complexity within single-step conversions for the collection of polycyclic skeletons. ${ }^{11}$ Such reactions feature bond forming efficiency and high levels of structural complexity while minimizing the generation of waste. ${ }^{12}$ In recent years, a great deal of effort has been devoted to carrying out MDRs for the assembly of various heterocycles. ${ }^{13}$ Over the past few years, we have also developed new multicomponent domino reactions of arylglyoxals with enaminones and different nucleophiles $(\mathrm{Nu})$, providing highly substituted indoles. ${ }^{14}$ Very recently, Shi and co-workers reported L-proline-catalyzed diastereoselective threecomponent reaction of acenaphthylene-1,2-dione, $\mathrm{N}$-aryl enaminones, and barbituric acid for the synthesis of functionalized tetrahydroacenaphtho[1,2-b]indolones. ${ }^{15}$ Later, Lin et al. represented a selective synthesis of acenaphtho[1,2b]indoles with different substitution patterns using acenaphthylene-1,2-dione and $N$-aryl enaminones as starting materials. The presence of $\mathrm{Et}_{3} \mathrm{~N}$ allowed a [3+2] cyclization to yield dihydroxylated acenaphtho[1,2-b]indoles whereas the use of $p$-toluenesulfonic acid ( $p$-TSA) led to alkoxylated analogue via cyclization and $S_{N} 1$-type reaction with alcohols (Scheme 1a). ${ }^{16}$ During this project, we believed that this reaction pathway may be adjusted by the use of suitable solvents or promoters to realize allylic hydroxylation of cyclic enaminones based on our previous studies. ${ }^{14}$ As we had expected, this transformation was successfully accomplished under microwave heating (MW) with concomitant achievement of selective allylic hydroxylation and esterification by controlling reaction temperatures (Schemes $1 \mathrm{~b}$ and $1 \mathrm{c})$. Further investigations revealed that replacing 5,5dimethylcyclohexane-1,3-dione-derived enaminones with 4,4dimethyl counterparts allowed formal $[3+2]$ cyclization to 
deliver pentacyclic-fused indoles 6 through dehydrogenation (Scheme 1d). Besides, mono-substituent such as a methyl or a phenyl group at 5-postion of cyclic enaminones was proven to be an effective substrate, transforming into the aromatic pentacyclic-fused indoles $\mathbf{8}$ through esterification (Scheme $1 \mathrm{e})$. Notably, purification of these pentacyclic-fused indoles could be achieved without traditional chromatography or recrystallization. Instead, products were isolated by diluting the reaction mixture with common solvents such as water or ethanol, and then collecting the precipitated solids by filtration. Such purifications characterize group-assisted purification (GAP) chemistry. ${ }^{17}$ In this paper, we report these interesting observations.
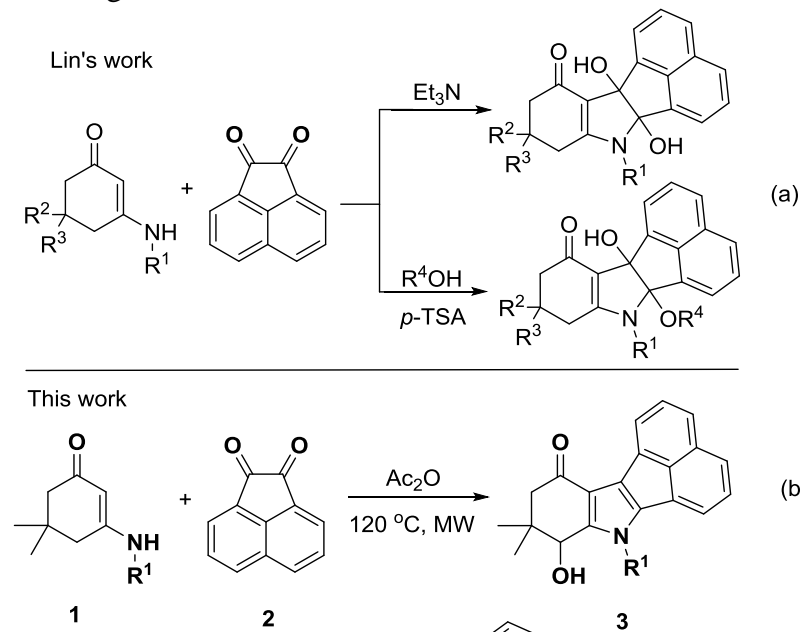

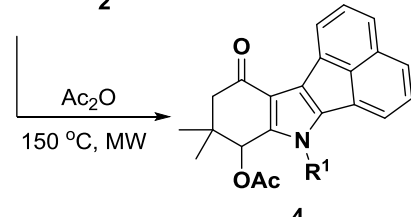

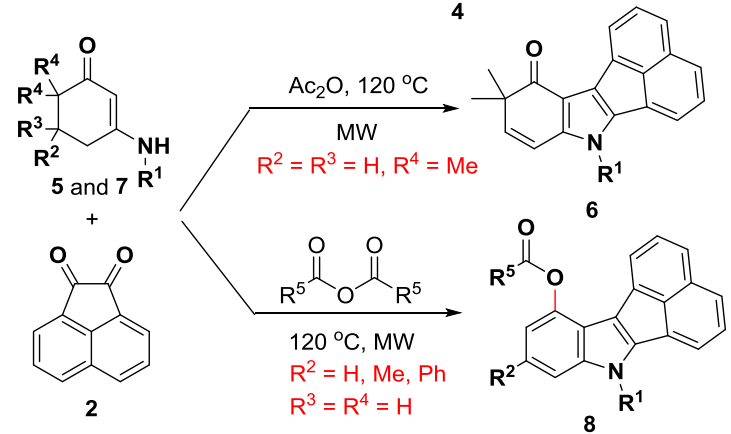

Scheme 1. Selective synthesis of pentacyclic indoles

\section{Results and discussion}

Initially, we performed the reaction of 3-((4chlorophenyl)amino)-5,5-dimethylcyclohex-2-enone (1a) with acenaphthylene-1,2-dione (2) in HOAc under microwave (MW) irradiation to evaluate the feasibility of allylic hydroxylation. The desired allylic hydroxylated product 3a was obtained, albeit with very low yield (15\%, Table 1, entry 1$)$. Subsequently, the reaction conditions were optimized to improve the yield of $\mathbf{3 a}$. The solvent effect was first investigated, and different solvents often used in organic synthesis, such as THF, MeCN, 1,4dioxane, TFA and DMF, were employed. Disappointedly, all these solvents failed to give the desired product 3a at $100{ }^{\circ} \mathrm{C}$ under microwave irradiation (entries 2-6). Considering the dehydration in cyclization process, we decided to use $\mathrm{Ac}_{2} \mathrm{O}$ as both a dehydrating agent and a solvent to improve the reaction efficiency. The formal $[3+2]$ cyclization in $\mathrm{Ac}_{2} \mathrm{O}^{18}$ worked efficiently to give product $3 \mathbf{a}$ with an isolated yield of $46 \%$ (entry 7). Next, we observed that the increase of the reaction temperature facilitated the reaction process. Elevating temperature to $110{ }^{\circ} \mathrm{C}$ led to a $65 \%$ yield (entry 8). The best result $(78 \%)$ was achieved when the temperature was enhanced to $120{ }^{\circ} \mathrm{C}$ (entry 9). Further increasing the reaction temperature is beneficial to form esterification product 4a. After careful optimizations, we found that the reaction at $120{ }^{\circ} \mathrm{C}$ preferred to give the allylic hydroxylated product $3 \mathbf{a}$ whereas allylic esterification product $\mathbf{4 a}$ was obtained in $81 \%$ chemical yield as the reaction was carried out at $150{ }^{\circ} \mathrm{C}$.

Table 1 Optimization Conditions for Forming $3 \mathbf{a}^{\mathrm{a}}$

\begin{tabular}{|c|c|c|c|c|}
\hline & $1 a$ & & & \\
\hline Entry & Solvent & $\mathrm{T} /{ }^{\circ} \mathrm{C}$ & Time/min & Yield $\mathrm{b} / \%$ \\
\hline 1 & HOAc & 100 & 22 & 15 \\
\hline 2 & THF & 100 & 22 & trace \\
\hline 3 & $\mathrm{MeCN}$ & 100 & 22 & trace \\
\hline 4 & 1,4-dioxane & 100 & 22 & trace \\
\hline 5 & TFA & 100 & 22 & trace \\
\hline 6 & DMF & 100 & 22 & trace \\
\hline 7 & $\mathrm{Ac}_{2} \mathrm{O}$ & 100 & 22 & 46 \\
\hline 8 & $\mathrm{Ac}_{2} \mathrm{O}$ & 110 & 22 & 65 \\
\hline 9 & $\mathrm{Ac}_{2} \mathrm{O}$ & 120 & 22 & 78 \\
\hline 10 & $\mathrm{Ac}_{2} \mathrm{O}$ & 130 & 22 & 56 \\
\hline 11 & $\mathrm{Ac}_{2} \mathrm{O}$ & 150 & 22 & trace \\
\hline
\end{tabular}

With the optimized conditions in hand, we then used various preformed $N$-substituted enaminones to explore the scope of allylic hydroxylation and esterification, respectively. The results are presented in Tables 2 and 3. Upon repeating the reaction with acenaphthylene-1,2-dione $(2)$ at $120{ }^{\circ} \mathrm{C}$, we are pleased to find that the formal $[3+2]$ cyclization reaction tolerated a broad spectrum of enaminones $\mathbf{1 a - 1 \mathbf { j }}$ bearing both electron-donating and electron-withdrawing groups, efficiently transformed into the corresponding polyfunctionalized pentacyclic-fused indoles $\mathbf{3 a - 3 j}$ with yields ranging from $72 \%$ to $85 \%$. The variant of substituents that resided at different positions on the phenyl ring, such as fluoro, chloro, bromo, methyl, and methoxy, was compatible (Table 2). After the successful realization of allylic hydroxylation, we next extended our investigation to the allylic esterification by adjusting reaction temperature to 150 ${ }^{\circ} \mathrm{C}$ under microwave heating. As per our expectation, a series of new esterified pentacyclic-fused indoles 4a-4o with 64\%$81 \%$ yields were provided in a highly selective and functional group-compatible manner under the optimized conditions. 
Table 2 Domino synthesis of pentacyclic indoles $\mathbf{3}$

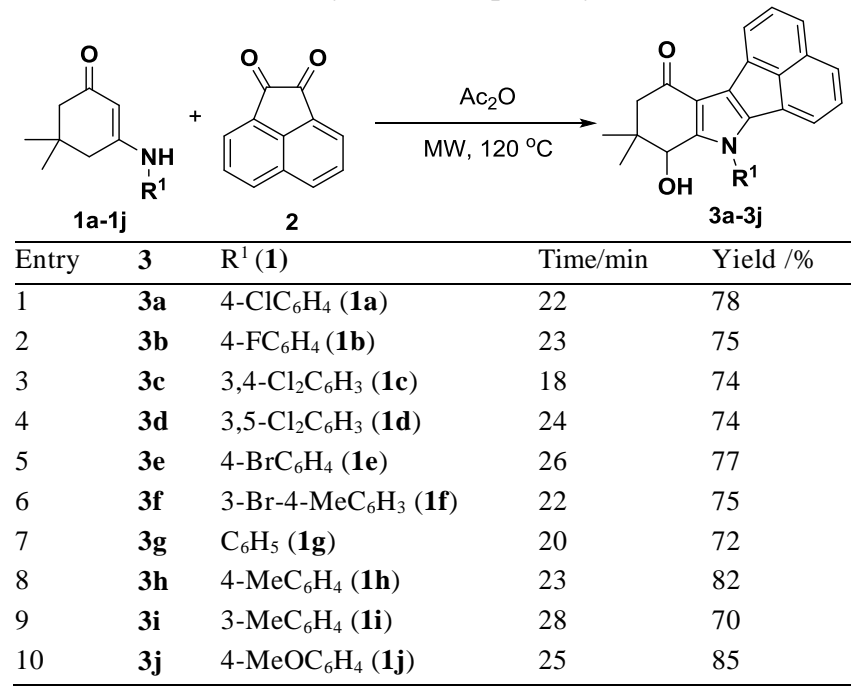

Table 3 Domino synthesis of pentacyclic indoles 4

\begin{tabular}{|c|c|c|c|c|c|}
\hline & $-1 m$ & 2 & & $\mathbf{R}^{5}$ & ta-4o \\
\hline Entry & 4 & $\mathrm{R}^{1}(\mathbf{1})$ & $\mathrm{R}^{5}$ & Time/min & Yield $1 \%$ \\
\hline 1 & $4 a$ & $4-\mathrm{ClC}_{6} \mathrm{H}_{4}(\mathbf{1 a})$ & $\mathrm{Me}$ & 20 & 81 \\
\hline 2 & 4b & $4-\mathrm{O}_{2} \mathrm{NC}_{6} \mathrm{H}_{4}(\mathbf{1 k})$ & $\mathrm{Me}$ & 29 & 66 \\
\hline 3 & $4 c$ & $2-\mathrm{MeC}_{6} \mathrm{H}_{4}(\mathbf{1 l})$ & $\mathrm{Me}$ & 28 & 67 \\
\hline 4 & $4 d$ & 4- $\mathrm{FC}_{6} \mathrm{H}_{4}(\mathbf{1 b})$ & Et & 23 & 70 \\
\hline 5 & $4 e$ & $3,4-\mathrm{Cl}_{2} \mathrm{C}_{6} \mathrm{H}_{3}(\mathbf{1 c})$ & Et & 22 & 78 \\
\hline 6 & $4 f$ & $3,5-\mathrm{Cl}_{2} \mathrm{C}_{6} \mathrm{H}_{3}(\mathbf{1 d})$ & Et & 25 & 71 \\
\hline 7 & $4 g$ & 4- $\mathrm{BrC}_{6} \mathrm{H}_{4}(\mathbf{1 e})$ & Et & 17 & 74 \\
\hline 8 & $4 h$ & $4-\mathrm{O}_{2} \mathrm{NC}_{6} \mathrm{H}_{4}(\mathbf{1 k})$ & Et & 26 & 69 \\
\hline 9 & $4 \mathbf{i}$ & $\mathrm{C}_{6} \mathrm{H}_{5}(\mathbf{1 g})$ & Et & 22 & 72 \\
\hline 10 & $4 j$ & $3-\mathrm{MeC}_{6} \mathrm{H}_{4}(\mathbf{1 i})$ & Et & 28 & 70 \\
\hline 11 & $4 k$ & $2-\mathrm{MeC}_{6} \mathrm{H}_{4}(\mathbf{1 1})$ & Et & 30 & 64 \\
\hline 12 & 41 & $4-\mathrm{MeOC}_{6} \mathrm{H}_{4}(\mathbf{1} \mathbf{j})$ & Et & 19 & 77 \\
\hline 13 & $4 m$ & 4-BrBn (1m) & Et & 26 & 71 \\
\hline 14 & $4 n$ & $\mathrm{C}_{6} \mathrm{H}_{5}(\mathbf{1 g})$ & $n-\operatorname{Pr}$ & 25 & 72 \\
\hline 15 & 40 & $4-\mathrm{MeOC}_{6} \mathrm{H}_{4}(\mathbf{1} \mathbf{j})$ & $i$-Pr & 28 & 68 \\
\hline
\end{tabular}

Various anhydrides, such as acetic anhydride, propionic andydride, butyric anhydride and isobutyric anhydride could be served as dehydration reagents, esterification reagents, and reaction as well as media Bronsted acid promoter for the allylic functionalization simultaneously. Different electronic nature of substituents enaminones $\mathbf{1}$ did not hamper the reaction process. A range of enaminones 1a-1m possessing either electron-deficient, electron-neutral, or electron-rich substituents at different positions on the aromatic ring were well-tolerated. Notably, the sterically more demanding orthosubstituted $\mathrm{N}$-aryl enaminone $\mathbf{1 l}$ was found to have no influence on the course of the reaction, with esterification product $\mathbf{4 c}$ afforded in $67 \%$ yield. Alternatively, $N$-benzyl enaminone $1 \mathrm{~m}$ can show high reactivity, generating the corresponding product $\mathbf{4 m}$ in $71 \%$ yield. Indeed, this protocol enabled the direct formations of $\mathrm{C}-\mathrm{O}$ bonds from allylic $\mathrm{C}-\mathrm{H}$ bonds without the use of any metal catalysts, giving new examples for selective synthesis of fused indoles with different substituted patterns in a step-economical fashion, depending on different reaction temperatures. The unequivocally structural elucidation was determined by their
NMR spectroscopic analysis. In cases of $\mathbf{3 j}$ and $\mathbf{4 f}$, their structures were further confirmed by X-ray diffraction analysis (Figures 1 and 2).

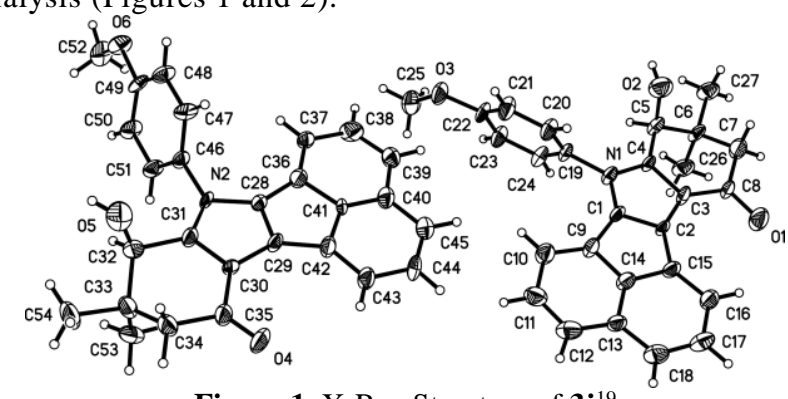

Figure 1. X-Ray Structure of $\mathbf{3} \mathbf{j}^{19}$

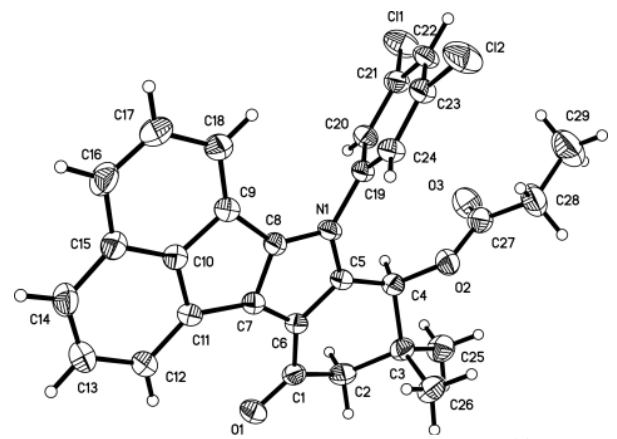

Figure 2. X-Ray Structure of $\mathbf{4 f}^{20}$

After successful formation of fused indoles $\mathbf{3}$ and $\mathbf{4}$, we turned our attention to investigating several differently substituted $N$ aryl enaminones to expand the synthetic utility of this methodology. We set out to use 6,6-dimethyl substituted enaminones 5 to replace 5,5-dimethyl analogue. The reaction was conducted at $120{ }^{\circ} \mathrm{C}$ under microwave heating, and 7 hydroxy fused indoles $\mathbf{4}$ were not obtained. Instead, dehydrogenated fused indoles $\mathbf{6}$ were afforded in good to excellent yields of 68\%-85\%. 4,4-Dimethyl substituted enaminones 5 carrying either electronically neutral, rich, or poor groups could be successfully engaged in the transformation (Scheme 2). Among them, halogen-containing enaminones 5 could be utilized and well-tolerated under the optimal conditions, delivering the expected products in good yields, which provide possible potential for further functionalizations by modern cross-coupling reactions. The structure of $\mathbf{6 d}$ was determined by X-ray diffraction analysis (Figure 3).

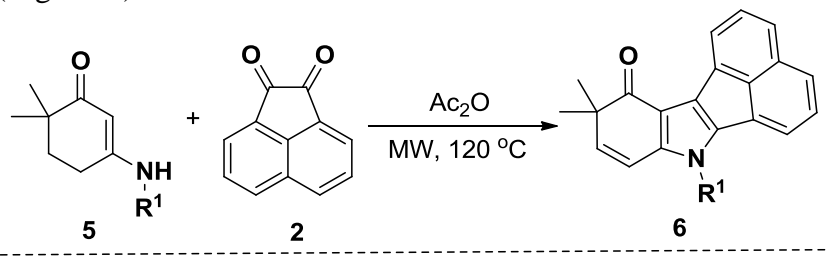

6a, $\mathrm{R}^{1}=4-\mathrm{ClC}_{6} \mathrm{H}_{4}(23 \mathrm{~min}, 77 \%) \quad 6 \mathbf{d}, \mathrm{R}^{1}=\mathrm{C}_{6} \mathrm{H}_{5}(20 \mathrm{~min}, 74 \%)$

6b, $\mathrm{R}^{1}=3,4-\mathrm{Cl}_{2} \mathrm{C}_{6} \mathrm{H}_{3}(20 \mathrm{~min}, 68 \%) 6 e, \mathrm{R}^{1}=4-\mathrm{MeC}_{6} \mathrm{H}_{4}(17 \mathrm{~min}, 85 \%)$ 6c, $\mathrm{R}^{1}=4-\mathrm{BrC}_{6} \mathrm{H}_{4}(19 \mathrm{~min}, 75 \%) \quad 6 f, \mathrm{R}^{1}=4-\mathrm{MeOC}_{6} \mathrm{H}_{4}(15 \mathrm{~min}, 80 \%)$

Scheme 2. Domino synthesis of pentacyclic indoles 6

On the basis of these observations described above, we believed that flexible structural modification of fused indole skeleton could be achieved by selectively introducing substituents at different positions of cyclic enaminone ring. Replacing 6,6dimethyl substituted enaminones 5 with 5-mono-substituted counterparts 7 further probed the reaction scope. The reaction proceeded smoothly to give access to a series of unprecedented pentacyclic indoles $\mathbf{8}$ in good yields through domino 
dehydrogenation, aromatization and esterification sequence under the optimal conditions described above. The results exhibit the wide scope and generality of this new multicomponent domino reaction with respect to various $N$-substituted enaminones with different electronic properties and anhydrides like acetic, propionic, butyric and isobutyric anhydrides (Scheme 3 ). The variation of substituents on cyclohexenone ring including methyl and phenyl groups all worked well for the present cyclization reaction and effectively converted into functionalized pentacyclic indoles 8. In addition to $N$-aryl substitutents, $N$ benzyl enaminone was also found to be a suitable substrate, affording the corresponding functionalized esterification products $\mathbf{8 h}$ and $\mathbf{8 p}$ in $66 \%$ and $67 \%$ yields, respectively. The structure of product 8e was based on X-ray diffraction analysis (Figure 4).
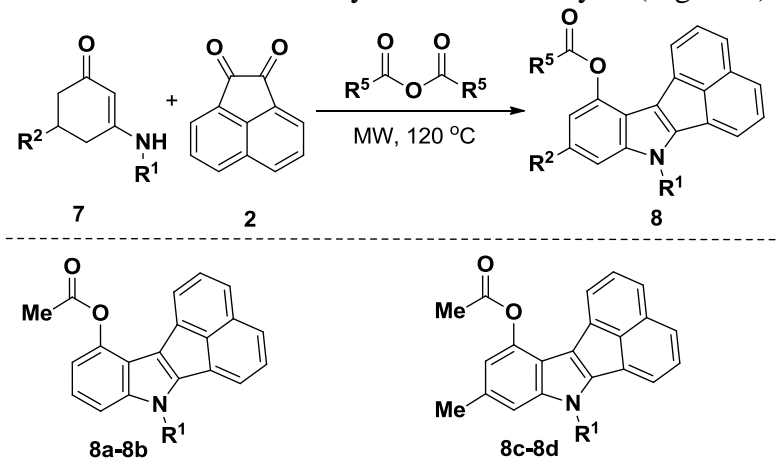

8a, $\mathrm{R}^{1}=\mathrm{C}_{6} \mathrm{H}_{5}(20 \mathrm{~min}, 73 \%)$

8b, $\mathrm{R}^{1}=4-\mathrm{ClC}_{6} \mathrm{H}_{4}(26 \mathrm{~min}, 76 \%)$

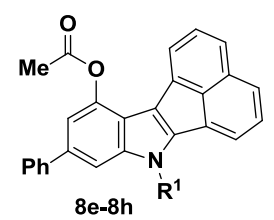

$8 e, \mathrm{R}^{1}=4-\mathrm{ClC}_{6} \mathrm{H}_{4}(17 \mathrm{~min}, 80 \%)$ 8f, $\mathrm{R}^{1}=4-\mathrm{BrC}_{6} \mathrm{H}_{4}(21 \mathrm{~min}, 77 \%)$ $8 \mathrm{~g}, \mathrm{R}^{1}=4-\mathrm{MeC}_{6} \mathrm{H}_{4}(22 \mathrm{~min}, 74 \%)$ $8 \mathrm{~h}, \mathrm{R}^{1}=\mathrm{Bn}(28 \mathrm{~min}, 66 \%)$

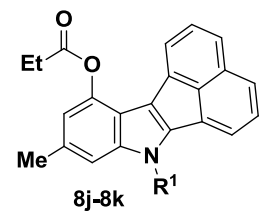

8j, $\mathrm{R}^{1}=4-\mathrm{BrC}_{6} \mathrm{H}_{4}$ (23 min, $\left.69 \%\right)$ 8k, $\mathrm{R}^{1}=4-\mathrm{MeC}_{6} \mathrm{H}_{4}(26 \mathrm{~min}, 85 \%)$

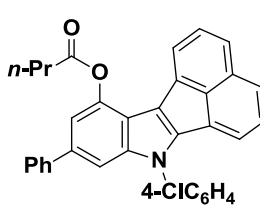

$8 q(25 \mathrm{~min}, 69 \%)$

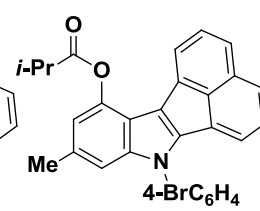

8 r (27 min, 70\%)

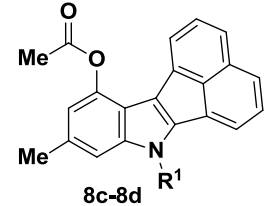

$8 c, \mathrm{R}^{1}=4-\mathrm{ClC}_{6} \mathrm{H}_{4}(18 \mathrm{~min}, 77 \%)$ 8d, $\mathrm{R}^{1}=4-\mathrm{MeOC}_{6} \mathrm{H}_{4}(15 \mathrm{~min}, 74 \%)$

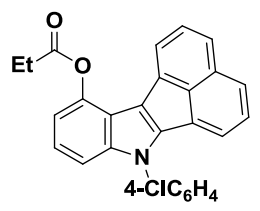

$8 \mathbf{i}(22 \mathrm{~min}, 71 \%)$

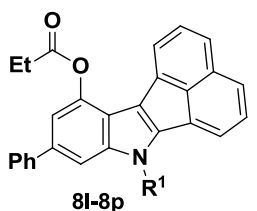

8I, $\mathrm{R}^{1}=4-\mathrm{ClC}_{6} \mathrm{H}_{4}$ (24 min, 74\%) $8 \mathrm{~m}, \mathrm{R}^{1}=3,5-\mathrm{Cl}_{2} \mathrm{C}_{6} \mathrm{H}_{3}(19 \mathrm{~min}, 75 \%)$ $8 \mathrm{n}, \mathrm{R}^{1}=4-\mathrm{BrC}_{6} \mathrm{H}_{4}$ (22 min, $\left.70 \%\right)$ 8o, $\mathrm{R}^{1}=4-\mathrm{MeC}_{6} \mathrm{H}_{4}(23 \mathrm{~min}, 78 \%)$ $8 p, R^{1}=B n(27 \min , 67 \%)$
Scheme 3. Domino synthesis of pentacycli indoles 8

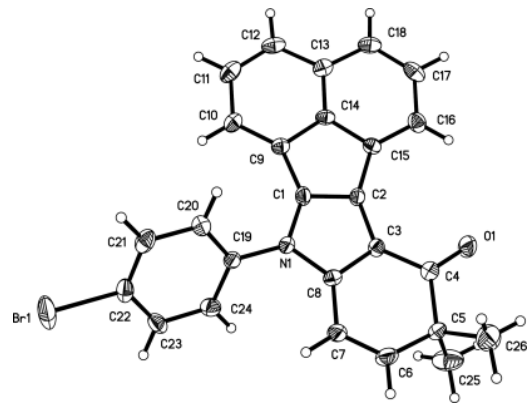

Figure 3. X-ray structure of $\mathbf{6} \mathbf{d}^{21}$

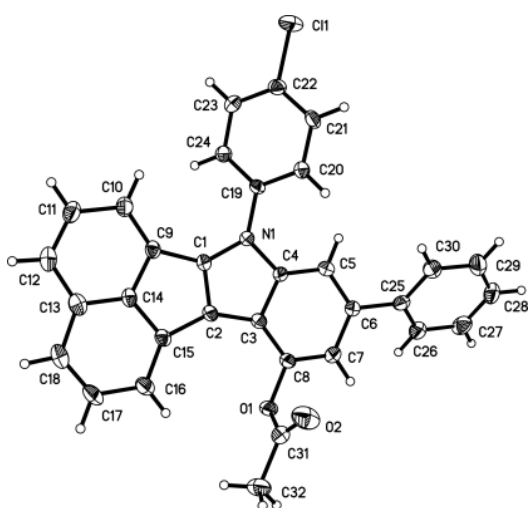

Figure 4. X-ray structure of $8 \mathbf{e}^{22}$

On the basis of the above observations and report survey, ${ }^{15,16}$ reasonable mechanisms for the selective formation of products $\mathbf{3}$, 4, 6 and 8 are proposed as shown in Scheme 5. The initial nucleophilic addition of enaminone $\mathbf{1}, \mathbf{5}$ and $\mathbf{7}$ to acenaphthylene1,2-dione $\mathbf{2}$ generates intermediates $\mathbf{A}$, followed by intramolecular cyclization and dehydration to gives intermediates C. With two methyl groups $\left(\mathrm{R}^{2}=\mathrm{R}^{3}=\mathrm{Me}\right)$ occupying $\mathrm{C5}$ position of cyclohexenone ring, intermediates $\mathbf{C}$ undergo protonation of hydroxy group and subsequent nucleophilic addition with water molecules, produce allylic hydroxylated fused indoles 3. At higher reaction temperature, products $\mathbf{3}$ are further transformed into fused indoles 4 through esterification with anhydrides. Similar to the former, when $\mathrm{R}^{3}$ is hydrogen and $\mathrm{R}^{4}$ is a methyl group, intermediates $\mathbf{C}$ undergo second dehydration to yield products $\mathbf{6}$ whereas the esterification indoles $\mathbf{7}$ are afforded through successive dehydration of intermediate $\mathbf{C}$ and esterification of intermediate $\mathbf{E}$ with both $\mathrm{R}^{3}$ and $\mathrm{R}^{4}$ being hydrogen.

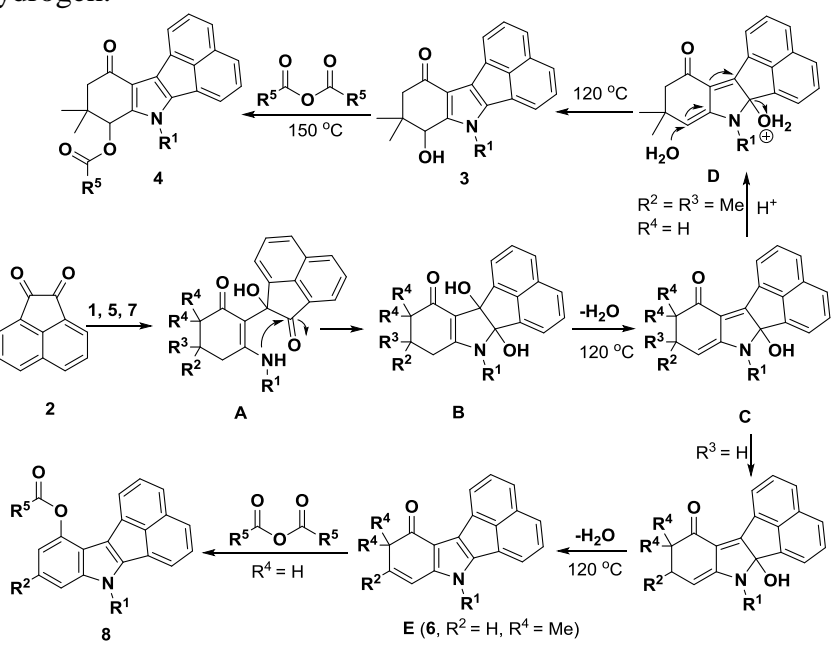

Scheme 5. Proposed mechanism for forming 3, 4, 6 and 8

\section{Conclusion}

In conclusion, we have described a new and practical multicomponent domino reactions ( $N$-substituted enaminones and acenaphthylene-1,2-dione) for selective synthesis of a broad range of new pentacyclic indoles with various functionalizations including allylic hydroxylation and esterification, depending on reaction temperatures and substituted patterns on cyclohexenone ring. These reactions feature convenient one-pot operation, short reaction times and flexible modification of fused indole skeleton. Additionally, the pure products could be obtained by using group-assisted purification (GAP) chemistry/ technology, avoiding traditional chromatography and recrystallization 
purification. The biological evaluation of the resulting pentacyclic indoles is underway in our lab.

\section{Experimental section}

Melting points were determined in open capillaries and were uncorrected. IR spectra were taken on a FT-IR-Tensor 27 spectrometer in $\mathrm{KBr}$ pellets and reported in $\mathrm{cm}^{-1}$. ${ }^{1} \mathrm{H}$ NMR $\left({ }^{13} \mathrm{C}\right.$ NMR) spectra were measured on a Bruker DPX $400 \mathrm{MHz}$ spectrometer in DMSO- $d_{6}$ with chemical shift $(\delta)$ given in ppm relative to TMS as internal standard $[(\mathrm{s}=$ singlet, $\mathrm{d}=$ doublet, $\mathrm{t}=$ triplet, brs $=$ broad singlet, $\mathrm{m}=$ multiplet), coupling constant $(\mathrm{Hz})]$. HRMS (ESI) was determined by using microTOF-QII HRMS/MS instrument (BRUKER). X-Ray crystallographic analysis was performed with a Siemens SMART CCD and a Siemens P4 diffractometer.

General procedure for the synthesis of compounds $3 \mathbf{a}-\mathbf{3 j}$ 7-(4-Chlorophenyl)-8-hydroxy-9,9-dimethyl-9,10-dihydro$7 H$-acenaphtho[1,2-b]indol-11 $(8 H)$-one (3a)

In a typical experiment procedure, 3-((4chlorophenyl)amino)-5,5-dimethylcyclohex-2-enone 1a (1.0 mmol, $0.249 \mathrm{~g}, 1.0$ equiv) and acenaphthylene-1,2-dione 2 $(1.0 \mathrm{mmol}, 0.182 \mathrm{~g}, 1.0$ equiv) were introduced in a $10-\mathrm{mL}$ Initiator $^{\mathrm{TM}}$ reaction vial, acetic anhydride $(1.5 \mathrm{~mL})$ was then successively added. Subsequently, the reaction vial was capped and then pre-stirring for 20 second. The mixture was irradiated (Time: $22 \mathrm{~min}$, Temperature: $120{ }^{\circ} \mathrm{C}$; Absorption Level: High; Fixed Hold Time) until TLC revealed that conversion of the starting materials were completed. The reaction mixture was then cooled to room temperature and then diluted with cold water $(25 \mathrm{~mL})$. The solid product was collected by Büchner filtration to give almost pure allylic hydroxylated indoles 3a, which was further purified by washing from $95 \%$ ethanol.

A yellow solid: $0.322 \mathrm{~g}$, yield $78 \%$; Mp: $226-228{ }^{\circ} \mathrm{C}$;

${ }^{1} \mathrm{H}$ NMR (400 MHz, DMSO- $\left.d_{6}\right)(\delta, \mathrm{ppm}): 8.04(\mathrm{~d}, 1 \mathrm{H}, J=6.8$ $\mathrm{Hz}, \mathrm{ArH}), 7.91(\mathrm{~d}, 2 \mathrm{H}, J=8.8 \mathrm{~Hz}, \operatorname{ArH}), 7.80(\mathrm{t}, 4 \mathrm{H}, J=7.6 \mathrm{~Hz}$, $\operatorname{ArH}), 7.62(\mathrm{t}, 1 \mathrm{H}, J=7.2 \mathrm{~Hz}, \operatorname{ArH}), 7.46(\mathrm{t}, 1 \mathrm{H}, J=7.6 \mathrm{~Hz}$, ArH), $7.20(\mathrm{~d}, 1 \mathrm{H}, J=7.2 \mathrm{~Hz}, \operatorname{ArH}), 5.68(\mathrm{~d}, 1 \mathrm{H}, J=7.6 \mathrm{~Hz}$, $\mathrm{CH}), 4.13(\mathrm{~d}, 1 \mathrm{H}, J=8.0 \mathrm{~Hz}, \mathrm{OH}), 2.86(\mathrm{~d}, 1 \mathrm{H}, J=16.4 \mathrm{~Hz}$, $\mathrm{CH}), 2.16(\mathrm{~d}, 1 \mathrm{H}, J=16.4 \mathrm{~Hz}, \mathrm{CH}), 1.11\left(\mathrm{~s}, 3 \mathrm{H}, \mathrm{CH}_{3}\right), 0.98(\mathrm{~s}$, $\left.3 \mathrm{H}, \mathrm{CH}_{3}\right)$;

${ }^{13} \mathrm{C}$ NMR $\left(100 \mathrm{MHz}, \mathrm{DMSO}-d_{6}\right)(\delta, \mathrm{ppm}): 193.7,147.9,139.4$, $136.1,133.9,131.7,130.5,129.5,128.6,128.5,128.2,127.9$, 127.2, 126.9, 125.0, 123.6, 120.0, 116.1, 68.3, 47.4, 26.1, 26.0 ;

IR $\left(\mathrm{KBr}, v, \mathrm{~cm}^{-1}\right): 3442,3068,1678,1647,1523,1496,1456$, 1432, 1403, 1381, 1347;

HRMS (ESI): m/z calcd for: $\mathrm{C}_{26} \mathrm{H}_{19} \mathrm{ClNO}_{2}, 412.1105[\mathrm{M}-\mathrm{H}]^{-}$; found: 412.1116 .

7-(4-Fluorophenyl)-8-hydroxy-9,9-dimethyl-9,10-dihydro-7Hacenaphtho[1,2-b]indol-11(8H)-one (3b)

A yellow solid: $0.298 \mathrm{~g}$, yield $75 \%$; Mp: $215-217^{\circ} \mathrm{C}$;

${ }^{1} \mathrm{H}$ NMR $\left(400 \mathrm{MHz}, \mathrm{DMSO}-d_{6}\right)(\delta, \mathrm{ppm}): 8.03(\mathrm{~d}, 1 \mathrm{H}, J=6.8$ $\mathrm{Hz}, \mathrm{ArH}), 7.92-7.89$ (m, 2H, ArH), 7.79 (d, 2H, $J=8.4 \mathrm{~Hz}$, ArH), 7.63-7.60 (m, 1H, ArH), $7.55(\mathrm{t}, 2 \mathrm{H}, J=8.8 \mathrm{~Hz}, \mathrm{ArH})$, 7.45 (t, $1 \mathrm{H}, J=7.2 \mathrm{~Hz}, \mathrm{ArH}), 7.14(\mathrm{~d}, 1 \mathrm{H}, J=6.8 \mathrm{~Hz}, \mathrm{ArH}), 5.64$ $(\mathrm{d}, 1 \mathrm{H}, J=7.6 \mathrm{~Hz}, \mathrm{CH}), 4.10(\mathrm{~d}, 1 \mathrm{H}, J=7.6 \mathrm{~Hz}, \mathrm{OH}), 2.86(\mathrm{~d}$, $1 \mathrm{H}, J=16.4 \mathrm{~Hz}, \mathrm{CH}), 2.15(\mathrm{~d}, 1 \mathrm{H}, J=16.4 \mathrm{~Hz}, \mathrm{CH}), 1.10$ (s, 3H, $\left.\mathrm{CH}_{3}\right), 0.98\left(\mathrm{~s}, 3 \mathrm{H}, \mathrm{CH}_{3}\right)$;

${ }^{13} \mathrm{C}$ NMR $\left(100 \mathrm{MHz}, \mathrm{DMSO}-d_{6}\right)(\delta, \mathrm{ppm}): 193.7,162.4\left({ }^{1} J_{\mathrm{CF}}=\right.$ $244.5 \mathrm{~Hz}), 148.0,139.6,133.5\left({ }^{4} J_{\mathrm{CF}}=2.8 \mathrm{~Hz}\right), 131.8\left({ }^{3} J_{\mathrm{CF}}=7.7\right.$ Hz), 129.5, 128.7, 128.6(3), 128.6(1), 128.5(8), 127.8, 127.2, $126.8,124.8,123.5,119.9,117.3\left({ }^{2} J_{\mathrm{CF}}=22.8 \mathrm{~Hz}\right), 115.9,68.3$, $47.4,26.2,26.0$
IR $\left(\mathrm{KBr}, v, \mathrm{~cm}^{-1}\right): 3431,3083,1726,1650,1608,1513,1481$, 1458, 1427, 1402, 1347, 1320;

HRMS (ESI): $\mathrm{m} / \mathrm{z}$ calcd for: $\mathrm{C}_{26} \mathrm{H}_{19} \mathrm{FNO}_{2}, 396.1400[\mathrm{M}-\mathrm{H}]^{-}$; found: 396.1412 .

7-(3,4-Dichlorophenyl)-8-hydroxy-9,9-dimethyl-9,10-dihydro7H-acenaphtho[1,2-b]indol-11 $(8 H)$-one $(3 \mathrm{c})$

A yellow solid: $0.331 \mathrm{~g}$, yield $74 \%$; Mp: $168-170{ }^{\circ} \mathrm{C}$

${ }^{1} \mathrm{H}$ NMR (400 MHz, DMSO- $\left.d_{6}\right)(\delta, \mathrm{ppm}): 8.24$ (s, 1H, ArH), 8.04 $(\mathrm{d}, 1 \mathrm{H}, J=6.8 \mathrm{~Hz}, \operatorname{ArH}), 7.99(\mathrm{~d}, 1 \mathrm{H}, J=8.4 \mathrm{~Hz}, \operatorname{ArH}), 7.91(\mathrm{~d}$, $1 \mathrm{H}, J=7.2 \mathrm{~Hz}, \operatorname{ArH}), 7.82(\mathrm{~d}, 2 \mathrm{H}, J=8.0 \mathrm{~Hz}, \operatorname{ArH}), 7.63(\mathrm{t}, 1 \mathrm{H}$, $J=7.6 \mathrm{~Hz}, \mathrm{ArH}), 7.48(\mathrm{t}, 1 \mathrm{H}, J=7.2 \mathrm{~Hz}, \mathrm{ArH}), 7.24(\mathrm{~d}, 1 \mathrm{H}, J=$ $6.8 \mathrm{~Hz}, \mathrm{ArH}), 5.72(\mathrm{~d}, 1 \mathrm{H}, J=7.6 \mathrm{~Hz}, \mathrm{CH}), 4.19(\mathrm{~d}, 1 \mathrm{H}, J=7.6$ $\mathrm{Hz}, \mathrm{OH}), 2.84(\mathrm{~d}, 1 \mathrm{H}, J=16.8 \mathrm{~Hz}, \mathrm{CH}), 2.18(\mathrm{~d}, 1 \mathrm{H}, J=16.0$ $\mathrm{Hz}, \mathrm{CH}), 1.11\left(\mathrm{~s}, 3 \mathrm{H}, \mathrm{CH}_{3}\right), 0.99$ (s, 3H, $\left.\mathrm{CH}_{3}\right)$;

${ }^{13} \mathrm{C}$ NMR $\left(100 \mathrm{MHz}, \mathrm{DMSO}-d_{6}\right)(\delta, \mathrm{ppm}): 193.8,147.9,139.3$, $137.1,132.7,132.3,132.2,131.7,131.6,129.5,128.6,128.4$, $128.3,127.9,127.3,127.0,126.8,125.2,123.7,120.1,116.3$, 68.3, 47.5, 26.0, 25.9;

IR $\left(\mathrm{KBr}, v, \mathrm{~cm}^{-1}\right): 3420,3071,1750,1726,1651,1606,1594$, $1525,1480,1458,1429,1349,1317$;

HRMS (ESI): m/z calcd for: $\mathrm{C}_{26} \mathrm{H}_{18} \mathrm{Cl}_{2} \mathrm{NO}_{2}, 446.0715$ [M-H]'; found: 446.0704 .

7-(3,5-Dichlorophenyl)-8-hydroxy-9,9-dimethyl-9,10-dihydro7H-acenaphtho[1,2-b]indol-11(8H)-one (3d)

A yellow solid: $0.331 \mathrm{~g}$, yield $74 \%$; Mp: $250-252{ }^{\circ} \mathrm{C}$;

${ }^{1} \mathrm{H}$ NMR (400 MHz, DMSO- $\left.d_{6}\right)(\delta, \mathrm{ppm}): 8.06-8.03(\mathrm{~m}, 3 \mathrm{H}$, ArH), $7.93(\mathrm{~s}, 1 \mathrm{H}, \operatorname{ArH}), 7.82(\mathrm{~d}, 2 \mathrm{H}, J=8.0 \mathrm{~Hz}, \operatorname{ArH}), 7.63(\mathrm{t}$, $1 \mathrm{H}, J=7.2 \mathrm{~Hz}, \mathrm{ArH}), 7.49(\mathrm{t}, 1 \mathrm{H}, J=7.6 \mathrm{~Hz}, \mathrm{ArH}), 7.22(\mathrm{~d}, 1 \mathrm{H}$, $J=7.2 \mathrm{~Hz}, \mathrm{ArH}), 5.75(\mathrm{~d}, 1 \mathrm{H}, J=7.6 \mathrm{~Hz}, \mathrm{CH}), 4.21(\mathrm{~d}, 1 \mathrm{H}, J=$ $7.6 \mathrm{~Hz}, \mathrm{OH}), 2.84$ (d, 1H, $J=16.4 \mathrm{~Hz}, \mathrm{CH}), 2.20$ (d, 1H, $J=16.4$ $\mathrm{Hz}, \mathrm{CH}), 1.12\left(\mathrm{~s}, 3 \mathrm{H}, \mathrm{CH}_{3}\right), 1.00\left(\mathrm{~s}, 3 \mathrm{H}, \mathrm{CH}_{3}\right)$;

${ }^{13} \mathrm{C}$ NMR (100 MHz, DMSO- $\left.d_{6}\right)(\delta, \mathrm{ppm}): 193.8,147.9,139.5$, $139.3,135.5,131.7,131.6,129.5,129.3,128.6,128.4,127.9$, 127.4, 127.0, 125.4, 125.3, 123.8, 119.9, 116.4, 68.4, 47.6, 26.1, 25.8 ;

IR $\left(\mathrm{KBr}, v, \mathrm{~cm}^{-1}\right): 3436,3080,1722,1655,1608,1587,1572$, 1526, 1480, 1459, 1434, 1387, 1348;

HRMS (ESI): $\mathrm{m} / \mathrm{z}$ calcd for: $\mathrm{C}_{26} \mathrm{H}_{18} \mathrm{Cl}_{2} \mathrm{NO}_{2}, 446.0715$ [M-H]'; found: 446.0707.

7-(4-Bromophenyl)-8-hydroxy-9,9-dimethyl-9,10-dihydro-7Hacenaphtho[1,2-b]indol-11 $(8 H)$-one $(3 \mathrm{e})$

A yellow solid: $0.352 \mathrm{~g}$, yield $77 \%$; Mp: $224-225^{\circ} \mathrm{C}$;

${ }^{1} \mathrm{H}$ NMR (400 MHz, DMSO- $\left.d_{6}\right)(\delta, \mathrm{ppm}): 8.04(\mathrm{~d}, 1 \mathrm{H}, J=6.8$ $\mathrm{Hz}, \operatorname{ArH}), 7.92(\mathrm{~d}, 2 \mathrm{H}, J=8.8 \mathrm{~Hz}, \operatorname{ArH}), 7.85-7.80(\mathrm{~m}, 4 \mathrm{H}$, ArH), $7.62(\mathrm{t}, 1 \mathrm{H}, J=7.2 \mathrm{~Hz}, \mathrm{ArH}), 7.46(\mathrm{t}, 1 \mathrm{H}, J=7.2 \mathrm{~Hz}$, $\operatorname{ArH}), 7.21(\mathrm{~d}, 1 \mathrm{H}, J=7.2 \mathrm{~Hz}, \operatorname{ArH}), 5.67(\mathrm{~d}, 1 \mathrm{H}, J=7.6 \mathrm{~Hz}$, $\mathrm{CH}), 4.13(\mathrm{~d}, 1 \mathrm{H}, J=7.6 \mathrm{~Hz}, \mathrm{OH}), 2.86(\mathrm{~d}, 1 \mathrm{H}, J=16.4 \mathrm{~Hz}$, $\mathrm{CH}), 2.16(\mathrm{~d}, 1 \mathrm{H}, J=16.4 \mathrm{~Hz}, \mathrm{CH}), 1.11\left(\mathrm{~s}, 3 \mathrm{H}, \mathrm{CH}_{3}\right), 0.98(\mathrm{~s}$, $\left.3 \mathrm{H}, \mathrm{CH}_{3}\right)$;

${ }^{13} \mathrm{C}$ NMR $\left(100 \mathrm{MHz}, \mathrm{DMSO}-d_{6}\right)(\delta, \mathrm{ppm}): 193.7,147.9,139.3$, $136.5,133.4,131.7,129.5,128.6,128.5,128.4,127.9,127.3$, 126.9, 125.1, 123.6, 122.4, 120.1, 116.1, 68.3, 47.4, 26.1, 26.0; IR $\left(\mathrm{KBr}, v, \mathrm{~cm}^{-1}\right): 3445,3068,1648,1524,1495,1456,1431$, 1418, 1402, 1379, 1366, 1346;

HRMS (ESI): $\mathrm{m} / \mathrm{z}$ calcd for: $\mathrm{C}_{26} \mathrm{H}_{19} \mathrm{BrNO}_{2}, 456.0599$ [M-H]'; found: 456.0608 .

7-(3-Bromo-4-methylphenyl)-8-hydroxy-9,9-dimethyl-9,10dihydro-7H-acenaphtho[1,2-b]indol-11(8H)-one (3f)

A yellow solid: $0.353 \mathrm{~g}$, yield $75 \%$; Mp: $214-216^{\circ} \mathrm{C}$;

${ }^{1} \mathrm{H}$ NMR (400 MHz, DMSO- $d_{6}$ ) $(\delta$, ppm): 8.12 (s, $1 \mathrm{H}, \mathrm{ArH}), 8.03$ $(\mathrm{d}, 1 \mathrm{H}, J=6.8 \mathrm{~Hz}, \mathrm{ArH}), 7.81(\mathrm{~d}, 3 \mathrm{H}, J=8.4 \mathrm{~Hz}, \mathrm{ArH}), 7.70(\mathrm{~d}$, $1 \mathrm{H}, J=8.0 \mathrm{~Hz}, \mathrm{ArH}), 7.62(\mathrm{t}, 1 \mathrm{H}, J=7.6 \mathrm{~Hz}, \mathrm{ArH}), 7.47(\mathrm{t}, 1 \mathrm{H}$, $J=7.6 \mathrm{~Hz}, \operatorname{ArH}), 7.17(\mathrm{~d}, 1 \mathrm{H}, J=7.2 \mathrm{~Hz}, \operatorname{ArH}), 5.67(\mathrm{~d}, 1 \mathrm{H}, J=$ 
$7.6 \mathrm{~Hz}, \mathrm{CH}), 4.12(\mathrm{~d}, 1 \mathrm{H}, J=7.6 \mathrm{~Hz}, \mathrm{OH}), 2.86(\mathrm{~d}, 1 \mathrm{H}, J=16.8$ $\mathrm{Hz}, \mathrm{CH}), 2.52\left(\mathrm{~s}, 3 \mathrm{H}, \mathrm{CH}_{3}\right), 2.15(\mathrm{~d}, 1 \mathrm{H}, J=16.4 \mathrm{~Hz}, \mathrm{CH}), 1.11$ (s, $\left.3 \mathrm{H}, \mathrm{CH}_{3}\right), 0.98\left(\mathrm{~s}, 3 \mathrm{H}, \mathrm{CH}_{3}\right)$;

${ }^{13} \mathrm{C}$ NMR (100 MHz, DMSO- $\left.d_{6}\right)(\delta, \mathrm{ppm}): 193.7,147.9,139.4$, $138.9, \quad 136.1, \quad 132.6,131.7,129.5(4), 129.5(1), 128.6(3)$, 128.5(7), 127.9, 127.2, 126.9, 125.6, 125.0, 124.9, 123.6, 119.8, 116.0, 68.4, 47.4, 26.1, 26.0, 22.7;

IR $\left(\mathrm{KBr}, v, \mathrm{~cm}^{-1}\right): 3351,3044,1656,1639,1603,1570,1521$, 1498, 1478, 1456, 1404, 1366, 1347;

HRMS (ESI): m/z calcd for: $\mathrm{C}_{27} \mathrm{H}_{21} \mathrm{BrNO}_{2}, 470.0756$ [M-H]; found: 470.0767.

\section{8-Hydroxy-9,9-dimethyl-7-phenyl-9,10-dihydro-7H- acenaphtho[1,2-b]indol-11(8H)-one $(3 \mathrm{~g})$}

A yellow solid: $0.273 \mathrm{~g}$, yield $72 \%$; Mp: $195-197{ }^{\circ} \mathrm{C}$;

${ }^{1} \mathrm{H}$ NMR (400 MHz, DMSO- $\left.d_{6}\right)(\delta, \mathrm{ppm}): 8.04(\mathrm{~d}, 1 \mathrm{H}, J=6.8$ $\mathrm{Hz}, \operatorname{ArH}), 7.86(\mathrm{~d}, 2 \mathrm{H}, J=7.2 \mathrm{~Hz}, \operatorname{ArH}), 7.80(\mathrm{~d}, 2 \mathrm{H}, J=8.0 \mathrm{~Hz}$, ArH), $7.72(\mathrm{t}, 2 \mathrm{H}, J=7.6 \mathrm{~Hz}, \mathrm{ArH}), 7.66-7.60(\mathrm{~m}, 2 \mathrm{H}, \mathrm{ArH})$, $7.44(\mathrm{t}, 1 \mathrm{H}, J=7.6 \mathrm{~Hz}, \mathrm{ArH}), 7.14(\mathrm{~d}, 1 \mathrm{H}, J=7.2 \mathrm{~Hz}, \mathrm{ArH}), 5.65$ $(\mathrm{d}, 1 \mathrm{H}, J=7.6 \mathrm{~Hz}, \mathrm{CH}), 4.11(\mathrm{~d}, 1 \mathrm{H}, J=7.6 \mathrm{~Hz}, \mathrm{OH}), 2.88(\mathrm{~d}$, $1 \mathrm{H}, J=16.4 \mathrm{~Hz}, \mathrm{CH}), 2.15(\mathrm{~d}, 1 \mathrm{H}, J=16.4 \mathrm{~Hz}, \mathrm{CH}), 1.11$ (s, 3H, $\left.\mathrm{CH}_{3}\right), 0.98$ (s, $\left.3 \mathrm{H}, \mathrm{CH}_{3}\right)$;

${ }^{13} \mathrm{C}$ NMR (100 MHz, DMSO- $\left.d_{6}\right)(\delta, \mathrm{ppm}): 193.7,148.0,139.5$, $137.2,131.9,131.8,130.4,129.6,129.5,128.8,128.6,127.8$, $127.1,126.7,126.3,124.9,123.5,119.8,116.0,68.4,47.3,26.3$, 26.0 ;

IR $\left(\mathrm{KBr}, v, \mathrm{~cm}^{-1}\right): 3432,3043,1715,1638,1594,1518,1458$, 1397, 1362, 1348;

HRMS (ESI): m/z calcd for: $\mathrm{C}_{26} \mathrm{H}_{20} \mathrm{NO}_{2}, 378.1494$ [M-H]'; found: 378.1482 .

\section{8-Hydroxy-9,9-dimethyl-7-( $p$-tolyl)-9,10-dihydro-7H- acenaphtho[1,2-b]indol-11(8H)-one (3h) \\ A yellow solid: $0.322 \mathrm{~g}$, yield $82 \%$; Mp: $194-196{ }^{\circ} \mathrm{C}$;}

${ }^{1} \mathrm{H}$ NMR (400 MHz, DMSO- $\left.d_{6}\right)(\delta, \mathrm{ppm}): 8.03(\mathrm{~d}, 1 \mathrm{H}, J=6.8$ $\mathrm{Hz}, \mathrm{ArH}), 7.80-7.77(\mathrm{~m}, 2 \mathrm{H}, \mathrm{ArH}), 7.73(\mathrm{~d}, 2 \mathrm{H}, J=8.0 \mathrm{~Hz}$, ArH), 7.63-7.59 (m, 1H, ArH), $7.51(\mathrm{~d}, 2 \mathrm{H}, J=8.4 \mathrm{~Hz}, \mathrm{ArH})$, 7.46-7.42 (m, 1H, ArH), $7.14(\mathrm{~d}, 1 \mathrm{H}, J=6.8 \mathrm{~Hz}, \operatorname{ArH}), 5.61(\mathrm{~d}$, $1 \mathrm{H}, J=7.6 \mathrm{~Hz}, \mathrm{CH}), 4.09(\mathrm{~d}, 1 \mathrm{H}, J=7.6 \mathrm{~Hz}, \mathrm{OH}), 2.87(\mathrm{~d}, 1 \mathrm{H}, J$ $=16.4 \mathrm{~Hz}, \mathrm{CH}), 2.48\left(\mathrm{~s}, 3 \mathrm{H}, \mathrm{CH}_{3}\right), 2.13(\mathrm{~d}, 1 \mathrm{H}, J=16.8 \mathrm{~Hz}$, $\mathrm{CH}), 1.11\left(\mathrm{~s}, 3 \mathrm{H}, \mathrm{CH}_{3}\right), 0.97\left(\mathrm{~s}, 3 \mathrm{H}, \mathrm{CH}_{3}\right)$;

${ }^{13} \mathrm{C}$ NMR (100 MHz, DMSO-d $)(\delta, \mathrm{ppm}): 193.7,148.0,139.5$, 139.1, 134.6, 131.9, 131.8, 130.8, 129.5, 128.8, 128.6, 127.8, $127.1,126.7,126.1,124.7,123.4,119.8,115.8,68.4,47.3,26.3$, 25.9, 21.3;

IR $\left(\mathrm{KBr}, v, \mathrm{~cm}^{-1}\right): 3441,3067,1648,1607,1516,1482,1456$, 1433, 1390, 1380, 1366, 1346;

HRMS (ESI): m/z calcd for: $\mathrm{C}_{27} \mathrm{H}_{22} \mathrm{NO}_{2}, 392.1651$ [M-H]'; found: 392.1665 .

\section{8-Hydroxy-9,9-dimethyl-7-( $m$-tolyl)-9,10-dihydro-7H- acenaphtho[1,2-b]indol-11 $(8 H)$-one $(3 i)$}

A yellow solid: $0.275 \mathrm{~g}$, yield $70 \%$; Mp: $165-167^{\circ} \mathrm{C}$;

${ }^{1} \mathrm{H}$ NMR (400 MHz, DMSO- $\left.d_{6}\right)(\delta, \mathrm{ppm}): 8.03(\mathrm{~d}, 1 \mathrm{H}, J=6.8$ $\mathrm{Hz}, \mathrm{ArH}), 7.80-7.78$ (m, 2H, ArH), 7.67-7.57 (m, 4H, ArH), 7.44 (t, $2 \mathrm{H}, J=7.6 \mathrm{~Hz}, \mathrm{ArH}), 7.14(\mathrm{~d}, 1 \mathrm{H}, J=6.8 \mathrm{~Hz}, \mathrm{ArH}), 5.64(\mathrm{~d}$, $1 \mathrm{H}, J=7.6 \mathrm{~Hz}, \mathrm{CH}), 4.11(\mathrm{~d}, 1 \mathrm{H}, J=7.6 \mathrm{~Hz}, \mathrm{OH}), 2.87(\mathrm{~d}, 1 \mathrm{H}, J$ $=16.4 \mathrm{~Hz}, \mathrm{CH}), 2.49\left(\mathrm{~s}, 3 \mathrm{H}, \mathrm{CH}_{3}\right), 2.14(\mathrm{~d}, 1 \mathrm{H}, J=16.4 \mathrm{~Hz}$, $\mathrm{CH}), 1.11\left(\mathrm{~s}, 3 \mathrm{H}, \mathrm{CH}_{3}\right), 0.98\left(\mathrm{~s}, 3 \mathrm{H}, \mathrm{CH}_{3}\right)$;

${ }^{13} \mathrm{C}$ NMR (100 MHz, DMSO-d $)(\delta, \mathrm{ppm}): 193.7,147.9,140.1$, $139.4,137.1,131.9,131.8,130.2,130.1,129.5,128.8,128.6$, $127.8,127.1,126.7,126.6,124.8,123.4,123.3,119.9,115.9$, 68.3, 47.3, 26.3, 25.9, 21.4;

IR $\left(\mathrm{KBr}, v, \mathrm{~cm}^{-1}\right): 3433,3043,1653,1609,1591,1523,1493$, 1478, 1456, 1398, 1376, 1346;
HRMS (ESI): m/z calcd for: $\mathrm{C}_{27} \mathrm{H}_{22} \mathrm{NO}_{2}, 392.1651$ [M-H]; found: 392.1662 .

\section{8-Hydroxy-7-(4-methoxyphenyl)-9,9-dimethyl-9,10-dihydro-}

$7 H$-acenaphtho[1,2-b]indol-11 $(8 H)$-one $(3 \mathbf{j})$

A yellow solid: $0.348 \mathrm{~g}$, yield $85 \%$; Mp: $259-261{ }^{\circ} \mathrm{C}$;

${ }^{1} \mathrm{H}$ NMR (400 MHz, DMSO- $d_{6}$ ) $(\delta, \mathrm{ppm}): 8.02(\mathrm{~d}, 1 \mathrm{H}, J=6.8$ $\mathrm{Hz}, \mathrm{ArH}), 7.77$ (t, $4 \mathrm{H}, J=8.4 \mathrm{~Hz}, \mathrm{ArH}), 7.63-7.59(\mathrm{~m}, 1 \mathrm{H}, \mathrm{ArH})$, $7.44(\mathrm{t}, 1 \mathrm{H}, J=7.2 \mathrm{~Hz}, \mathrm{ArH}), 7.24$ (d, 2H, $J=9.2 \mathrm{~Hz}, \mathrm{ArH}), 7.13$ $(\mathrm{d}, 1 \mathrm{H}, J=6.8 \mathrm{~Hz}, \mathrm{ArH}), 5.60(\mathrm{~d}, 1 \mathrm{H}, J=7.6 \mathrm{~Hz}, \mathrm{CH}), 4.07(\mathrm{~d}$, $1 \mathrm{H}, J=7.6 \mathrm{~Hz}, \mathrm{OH}), 3.91\left(\mathrm{~s}, 3 \mathrm{H}, \mathrm{OCH}_{3}\right), 2.87(\mathrm{~d}, 1 \mathrm{H}, J=16.4$ $\mathrm{Hz}, \mathrm{CH}), 2.13(\mathrm{~d}, 1 \mathrm{H}, J=16.8 \mathrm{~Hz}, \mathrm{CH}), 1.11\left(\mathrm{~s}, 3 \mathrm{H}, \mathrm{CH}_{3}\right), 0.97$ (s, $3 \mathrm{H}, \mathrm{CH}_{3}$ );

${ }^{13} \mathrm{C}$ NMR (100 MHz, DMSO- $\left.d_{6}\right)(\delta, \mathrm{ppm}): 193.7,159.9,148.1$, $139.7,132.0,131.8,129.8,129.5,128.8,128.6,127.8,127.7$, $127.0,126.6,124.5,123.4,119.8,115.7,115.4,68.3,56.0,47.3$, 26.3, 25.9;

IR $\left(\mathrm{KBr}, v, \mathrm{~cm}^{-1}\right): 3336,3055,1644,1612,1513,1459,1438$, 1380, 1346;

HRMS (ESI): $\mathrm{m} / \mathrm{z}$ calcd for: $\mathrm{C}_{27} \mathrm{H}_{22} \mathrm{NO}_{3}, 408.1600 \quad[\mathrm{M}-\mathrm{H}]^{-}$; found: 408.1591 .

\section{General Procedure for the Synthesis of 4a-4o}

7-(4-Chlorophenyl)-9,9-dimethyl-11-oxo-8,9,10,11-

tetrahydro- $7 \mathrm{H}$-acenaphtho[1,2-b]indol-8-yl acetate $(4 a)$

In a typical experiment procedure, 3-((4-chlorophenyl)amino)5,5-dimethylcyclohex-2-enone $1 \mathbf{1 a}(1.0 \mathrm{mmol}, 0.249 \mathrm{~g}, 1.0$ equiv) and acenaphthylene-1,2-dione 2 (1.0 mmol, $0.182 \mathrm{~g}, 1.0$ equiv) were introduced in a $10-\mathrm{mL}$ Initiator ${ }^{\mathrm{TM}}$ reaction vial, acetic anhydride $(1.5 \mathrm{~mL})$ was then successively added. Subsequently, the reaction vial was capped and then pre-stirring for 20 second. The mixture was irradiated (Time: $20 \mathrm{~min}$, Temperature: $150{ }^{\circ} \mathrm{C}$; Absorption Level: High; Fixed Hold Time) until TLC revealed that conversion of the starting materials were completed. The reaction mixture was then cooled to room temperature and then diluted with cold water $(25 \mathrm{~mL})$. The solid product was collected by Büchner filtration to give almost pure allylic esterified indoles 4a, which was further purified by washing from $95 \%$ ethanol. A yellow solid: $0.369 \mathrm{~g}$, yield $81 \%$; Mp: $205-207{ }^{\circ} \mathrm{C}$;

${ }^{1} \mathrm{H}$ NMR (400 MHz, DMSO- $d_{6}$ ) $(\delta, \mathrm{ppm}): 8.05(\mathrm{~d}, 1 \mathrm{H}, J=6.4$ $\mathrm{Hz}, \mathrm{ArH}), 7.83$ (d, 2H, $J=8.0 \mathrm{~Hz}, \mathrm{ArH}), 7.75$ (d, 2H, $J=8.4 \mathrm{~Hz}$, ArH), 7.70 (d, 2H, $J=8.0 \mathrm{~Hz}, \operatorname{ArH}), 7.64(\mathrm{t}, 1 \mathrm{H}, J=7.6 \mathrm{~Hz}$, $\operatorname{ArH}), 7.45(\mathrm{t}, 1 \mathrm{H}, J=7.2 \mathrm{~Hz}, \operatorname{ArH}), 7.04(\mathrm{~d}, 1 \mathrm{H}, J=6.8 \mathrm{~Hz}$, ArH), 5.85 (s, 1H, CH), 2.74 (d, 1H, J = $16.8 \mathrm{~Hz}, \mathrm{CH}), 2.39$ (d, $1 \mathrm{H}, J=16.4 \mathrm{~Hz}, \mathrm{CH}), 1.91\left(\mathrm{~s}, 3 \mathrm{H}, \mathrm{CH}_{3}\right), 1.14\left(\mathrm{~s}, 3 \mathrm{H}, \mathrm{CH}_{3}\right), 1.02$ (s, $3 \mathrm{H}, \mathrm{CH}_{3}$ );

${ }^{13} \mathrm{C}$ NMR (100 MHz, DMSO- $\left.d_{6}\right)(\delta, \mathrm{ppm}): 192.8,169.8,142.6$, $140.8,135.6,134.6,131.7,131.4,130.6,129.6,128.9,128.7$, 128.0, 127.9, 127.6, 127.0, 124.5, 123.7, 120.1, 117.5, 69.4, 48.4, 26.1, 25.0, 20.7;

IR $\left(\mathrm{KBr}, v, \mathrm{~cm}^{-1}\right): 3057,1754,1688,1568,1514,1522,1490$, 1387, 1359, 1320;

HRMS (ESI): $\mathrm{m} / \mathrm{z}$ calcd for: $\mathrm{C}_{28} \mathrm{H}_{22} \mathrm{ClNNaO}_{3}, 478.1186$ $[\mathrm{M}+\mathrm{Na}]^{+}$; found: 478.1197 .

9,9-Dimethyl-7-(4-nitrophenyl)-11-oxo-8,9,10,11-tetrahydro$7 \mathrm{H}$-acenaphtho[1,2-b]indol-8-yl acetate $(\mathbf{4 b})$

A yellow solid: $0.308 \mathrm{~g}$, yield $66 \%$; Mp: $220-222^{\circ} \mathrm{C}$;

${ }^{1} \mathrm{H}$ NMR (400 MHz, DMSO- $d_{6}$ ) $(\delta, \mathrm{ppm}): 8.52(\mathrm{~d}, 2 \mathrm{H}, J=8.8$ $\mathrm{Hz}, \mathrm{ArH}), 8.08(\mathrm{~d}, 1 \mathrm{H}, J=6.8 \mathrm{~Hz}, \operatorname{ArH}), 8.00(\mathrm{~d}, 2 \mathrm{H}, J=8.8 \mathrm{~Hz}$, ArH), $7.85(\mathrm{~d}, 2 \mathrm{H}, J=8.4 \mathrm{~Hz}, \mathrm{ArH}), 7.66(\mathrm{t}, 1 \mathrm{H}, J=6.8 \mathrm{~Hz}$, $\operatorname{ArH}), 7.45(\mathrm{t}, 1 \mathrm{H}, J=7.6 \mathrm{~Hz}, \operatorname{ArH}), 7.15(\mathrm{~d}, 1 \mathrm{H}, J=7.2 \mathrm{~Hz}$, $\mathrm{ArH}), 5.92(\mathrm{~s}, 1 \mathrm{H}, \mathrm{CH}), 2.76(\mathrm{~d}, 1 \mathrm{H}, J=16.4 \mathrm{~Hz}, \mathrm{CH}), 2.43(\mathrm{~d}$, $1 \mathrm{H}, J=16.8 \mathrm{~Hz}, \mathrm{CH}), 1.88\left(\mathrm{~s}, 3 \mathrm{H}, \mathrm{CH}_{3}\right), 1.16\left(\mathrm{~s}, 3 \mathrm{H}, \mathrm{CH}_{3}\right), 1.03$ (s, 3H, $\left.\mathrm{CH}_{3}\right)$; 
${ }^{13} \mathrm{C}$ NMR $\left(100 \mathrm{MHz}, \mathrm{DMSO}-d_{6}\right)(\delta, \mathrm{ppm}): 192.9,169.9,148.1$, $142.5,142.2,140.5,131.7,131.2,129.6,128.7,128.4,127.9$, $127.8,127.3,127.2,126.0,125.0,124.0,120.5,118.0,69.5,48.4$, $26.1,24.9,20.6$

IR $\left(\mathrm{KBr}, v, \mathrm{~cm}^{-1}\right): 3069,1754,1693,1608,1573,1521,1481$, 1388, 1351, 1348;

HRMS (ESI): $\mathrm{m} / \mathrm{z}$ calcd for: $\mathrm{C}_{28} \mathrm{H}_{22} \mathrm{~N}_{2} \mathrm{NaO}_{5}, 489.1426[\mathrm{M}+\mathrm{Na}]^{+}$; found: 489.1411 .

\section{9,9-Dimethyl-11-oxo-7-(o-tolyl)-8,9,10,11-tetrahydro-7H- acenaphtho[1,2-b]indol-8-yl acetate $(4 \mathrm{c})$ \\ A yellow solid: $0.291 \mathrm{~g}$, yield $67 \%$; Mp: $165-167{ }^{\circ} \mathrm{C}$;}

${ }^{1} \mathrm{H}$ NMR $\left(400 \mathrm{MHz}, \mathrm{DMSO}-d_{6}\right)(\delta, \mathrm{ppm}): 8.04(\mathrm{~d}, 1 \mathrm{H}, J=7.2$ $\mathrm{Hz}, \mathrm{ArH})$, 7.81-7.77 (m, 2H, ArH), 7.65-7.61 (m, 1H, ArH), 7.57-7.54 (m, 2H, ArH), 7.48-7.45 (m, 2H, ArH), 7.40-7.36 (m, $1 \mathrm{H}, \operatorname{ArH}), 6.69(\mathrm{~d}, 1 \mathrm{H}, J=7.2 \mathrm{~Hz}, \operatorname{ArH}), 5.85+5.56(\mathrm{~s}, 1 \mathrm{H}$ $\mathrm{CH}), 2.79-2.67(\mathrm{~m}, 1 \mathrm{H}, \mathrm{CH}), 2.47-2.35(\mathrm{~m}, 1 \mathrm{H}, \mathrm{CH}), 2.06(\mathrm{~d}, 3 \mathrm{H}$, $\left.J=6.0 \mathrm{~Hz}, \mathrm{CH}_{3}\right), 1.94+1.75\left(\mathrm{~s}, 3 \mathrm{H}, \mathrm{CH}_{3}\right), 1.12(\mathrm{~d}, 3 \mathrm{H}, J=6.8$ $\left.\mathrm{Hz}, \mathrm{CH}_{3}\right), 1.00\left(\mathrm{~d}, 3 \mathrm{H}, J=5.6 \mathrm{~Hz}, \mathrm{CH}_{3}\right)$;

${ }^{13} \mathrm{C}$ NMR (100 MHz, DMSO- $\left.d_{6}\right)(\delta, \mathrm{ppm}): 192.7(1), 192.6(6)$, $169.6,169.3,142.5,142.4,140.9,140.7,135.8,135.5,135.2$, $132.0,131.8,131.7(2), 131.6(8), 130.6,130.5,129.6,129.5$, $128.7,128.2(5), 128.1(8), 128.1,128.0(3), 127.9(9), 127.9(5)$, 127.9(1), 127.8, 127.4(3), 127.4(0), 126.8(3), 126.8(1), 124.1(2), 124.0(7), 123.6, 119.3, 119.1, 117.1, 117.0, 69.5, 69.4, 48.9, $48.3,26.3,26.0,24.9,24.4,20.8,20.2,17.2,17.1$;

IR $\left(\mathrm{KBr}, v, \mathrm{~cm}^{-1}\right): 3033,1764,1698,1656,1580,1478,1425$, $1398,1366,1347$

HRMS (ESI): $\mathrm{m} / \mathrm{z}$ calcd for: $\mathrm{C}_{29} \mathrm{H}_{25} \mathrm{NNaO}_{3}, 458.1732[\mathrm{M}+\mathrm{Na}]^{+}$; found: 458.1749 .

7-(4-Fluorophenyl)-9,9-dimethyl-11-oxo-8,9,10,11-tetrahydro$7 H$-acenaphtho[1,2-b]indol-8-yl propionate (4d)

A yellow solid: $0.317 \mathrm{~g}$, yield $70 \%$; Mp: $189-191^{\circ} \mathrm{C}$;

${ }^{1} \mathrm{H}$ NMR $\left(400 \mathrm{MHz}, \mathrm{DMSO}-d_{6}\right)(\delta, \mathrm{ppm}): 8.04(\mathrm{~d}, 1 \mathrm{H}, J=6.8$ $\mathrm{Hz}, \mathrm{ArH}), 7.82$ (d, 2H, $J=8.0 \mathrm{~Hz}, \mathrm{ArH}), 7.72$ (s, 2H, ArH), 7.64 $(\mathrm{t}, 1 \mathrm{H}, J=7.2 \mathrm{~Hz}, \mathrm{ArH}), 7.51(\mathrm{t}, 2 \mathrm{H}, J=8.0 \mathrm{~Hz}, \mathrm{ArH}), 7.43(\mathrm{t}$, $1 \mathrm{H}, J=7.2 \mathrm{~Hz}, \operatorname{ArH}), 6.99(\mathrm{~d}, 1 \mathrm{H}, J=6.8 \mathrm{~Hz}, \operatorname{ArH}), 5.90(\mathrm{~s}, 1 \mathrm{H}$, $\mathrm{CH}), 2.72(\mathrm{~d}, 1 \mathrm{H}, J=16.4 \mathrm{~Hz}, \mathrm{CH}), 2.40(\mathrm{~d}, 1 \mathrm{H}, J=16.4 \mathrm{~Hz}$, $\mathrm{CH}), 2.30-2.21(\mathrm{~m}, 1 \mathrm{H}, \mathrm{CH}), 2.16-2.06(\mathrm{~m}, 1 \mathrm{H}, \mathrm{CH}), 1.14(\mathrm{~s}, 3 \mathrm{H}$, $\mathrm{CH}_{3}$ ), 1.01 (s, 3H, $\left.\mathrm{CH}_{3}\right), 0.92$ (t, $3 \mathrm{H}, J=7.6 \mathrm{~Hz}, \mathrm{CH}_{3}$ );

${ }^{13} \mathrm{C}$ NMR $\left(100 \mathrm{MHz}, \mathrm{DMSO}-d_{6}\right)(\delta, \mathrm{ppm}): 192.8,173.0,162.7$ $\left({ }^{1} J_{\mathrm{CF}}=245.5 \mathrm{~Hz}\right), 142.7,141.0,133.1,131.7\left({ }^{2} J_{\mathrm{CF}}=25.5 \mathrm{~Hz}\right)$, $129.6,129.4,129.3\left({ }^{4} J_{\mathrm{CF}}=2.6 \mathrm{~Hz}\right), 128.6,128.1,127.8,127.5$, $126.9,124.3,123.7,119.9,117.6,117.4\left({ }^{3} J_{\mathrm{CF}}=5.7 \mathrm{~Hz}\right), 69.3$, 48.5, 27.0, 26.2, 24.9, 9.4;

IR $\left(\mathrm{KBr}, v, \mathrm{~cm}^{-1}\right): 3059,1735,1660,1614,1511,1480,1459$ 1416, 1390, 1370, 1361;

HRMS (ESI): m/z calcd for: $\mathrm{C}_{29} \mathrm{H}_{24} \mathrm{FNNaO}_{3}, 476.1638$ [M+Na] $]^{+}$; found: 476.1625 .

\section{7-(3,4-Dichlorophenyl)-9,9-dimethyl-11-oxo-8,9,10,11-} tetrahydro-7H-acenaphtho[1,2-b]indol-8-yl propionate (4e) A yellow solid: $0.392 \mathrm{~g}$, yield $78 \%$; Mp: $157-159^{\circ} \mathrm{C}$;

${ }^{1} \mathrm{H}$ NMR (400 MHz, DMSO- $\left.d_{6}\right)(\delta, \mathrm{ppm}): 8.05$ (d, 2H, $J=6.8$ $\mathrm{Hz}, \mathrm{ArH}), 7.95(\mathrm{~d}, 1 \mathrm{H}, J=8.8 \mathrm{~Hz}, \mathrm{ArH}), 7.83(\mathrm{~d}, 2 \mathrm{H}, J=8.0 \mathrm{~Hz}$, $\operatorname{ArH}), 7.74(\mathrm{~d}, 1 \mathrm{H}, J=7.2 \mathrm{~Hz}, \operatorname{ArH}), 7.64(\mathrm{t}, 1 \mathrm{H}, J=7.2 \mathrm{~Hz}$, ArH), $7.45(\mathrm{t}, 1 \mathrm{H}, J=7.6 \mathrm{~Hz}, \operatorname{ArH}), 7.11(\mathrm{~d}, 1 \mathrm{H}, J=6.8 \mathrm{~Hz}$, $\mathrm{ArH}), 5.93(\mathrm{~s}, 1 \mathrm{H}, \mathrm{CH}), 2.71(\mathrm{~d}, 1 \mathrm{H}, J=16.0 \mathrm{~Hz}, \mathrm{CH}), 2.43(\mathrm{~d}$, $1 \mathrm{H}, J=16.0 \mathrm{~Hz}, \mathrm{CH}), 2.28-2.04(\mathrm{~m}, 2 \mathrm{H}, \mathrm{CH}), 1.13\left(\mathrm{~s}, 3 \mathrm{H}, \mathrm{CH}_{3}\right)$, $1.01\left(\mathrm{~s}, 3 \mathrm{H}, \mathrm{CH}_{3}\right), 0.93$ (s, 3H, $\mathrm{CH}_{3}$ );

${ }^{13} \mathrm{C}$ NMR $\left(100 \mathrm{MHz}, \mathrm{DMSO}-d_{6}\right)(\delta, \mathrm{ppm}): 192.9,173.1,142.7$, $140.7,136.6,133.0,132.9,132.4,131.7,131.4,129.6,129.3$, $128.7,128.0,127.9,127.6,127.5,127.1,124.6,123.8,120.3$, $117.7,69.4,48.7,26.9,26.3,24.7,9.3$;

IR $\left(\mathrm{KBr}, v, \mathrm{~cm}^{-1}\right): 3065,1746,1666,1611,1593,1558,1518$, $1481,1458,1434,1390,1370$;
HRMS (ESI): $\mathrm{m} / \mathrm{z}$ calcd for: $\mathrm{C}_{29} \mathrm{H}_{23} \mathrm{Cl}_{2} \mathrm{NNaO}_{3}, \quad 526.0953$ $[\mathrm{M}+\mathrm{Na}]^{+}$; found: 526.0942 .

\section{7-(3,5-Dichlorophenyl)-9,9-dimethyl-11-oxo-8,9,10,11-} tetrahydro- $7 \mathrm{H}$-acenaphtho[1,2-b]indol-8-yl propionate (4f) A yellow solid: $0.357 \mathrm{~g}$, yield $71 \%$; $\mathrm{Mp}: 197-199^{\circ} \mathrm{C}$;

${ }^{1} \mathrm{H}$ NMR (400 MHz, DMSO- $\left.d_{6}\right)(\delta, \mathrm{ppm}): 8.05(\mathrm{~d}, 1 \mathrm{H}, J=6.8$ $\mathrm{Hz}, \mathrm{ArH}), 7.96-7.83(\mathrm{~m}, 5 \mathrm{H}, \mathrm{ArH}), 7.64(\mathrm{t}, 1 \mathrm{H}, J=7.2 \mathrm{~Hz}, \mathrm{ArH})$, $7.48(\mathrm{t}, 1 \mathrm{H}, J=7.6 \mathrm{~Hz}, \mathrm{ArH}), 7.09(\mathrm{~d}, 1 \mathrm{H}, J=6.8 \mathrm{~Hz}, \mathrm{ArH}), 5.92$ $(\mathrm{s}, 1 \mathrm{H}, \mathrm{CH}), 2.71(\mathrm{~d}, 1 \mathrm{H}, J=16.4 \mathrm{~Hz}, \mathrm{CH}), 2.42(\mathrm{~d}, 1 \mathrm{H}, J=16.0$ $\mathrm{Hz}, \mathrm{CH}), 2.35-2.25(\mathrm{~m}, 1 \mathrm{H}, \mathrm{CH}), 2.19-2.09$ (m, 1H, $\mathrm{CH}), 1.13$ (s, $\left.3 \mathrm{H}, \mathrm{CH}_{3}\right), 1.01\left(\mathrm{~s}, 3 \mathrm{H}, \mathrm{CH}_{3}\right), 0.96\left(\mathrm{t}, 3 \mathrm{H}, J=7.6 \mathrm{~Hz}, \mathrm{CH}_{3}\right)$;

${ }^{13} \mathrm{C}$ NMR (100 MHz, DMSO- $\left.d_{6}\right)(\delta, \mathrm{ppm}): 192.9,173.1,142.7$, $140.7,138.9,135.7,131.7,131.3,130.0,129.6,128.7,128.0$, $127.9,127.6,127.1,126.4,124.6,123.8,120.1,117.7,69.3,48.7$, 27.0, 26.3, 24.6, 9.3;

IR $\left(\mathrm{KBr}, v, \mathrm{~cm}^{-1}\right): 3045,1741,1666,1585,1571,1558,1524$, 1507, 1457, 1472, 1417, 1385;

HRMS (ESI): $\mathrm{m} / \mathrm{z}$ calcd for: $\mathrm{C}_{29} \mathrm{H}_{23} \mathrm{Cl}_{2} \mathrm{NNaO}_{3}, \quad 526.0953$ [M+Na] $]^{+}$; found: 526.0940 .

7-(4-Bromophenyl)-9,9-dimethyl-11-oxo-8,9,10,11tetrahydro-7H-acenaphtho[1,2-b]indol-8-yl propionate $(\mathbf{4 g})$ A yellow solid: $0.380 \mathrm{~g}$, yield $74 \%$; $\mathrm{Mp}: 155-157^{\circ} \mathrm{C}$;

${ }^{1} \mathrm{H}$ NMR $\left(400 \mathrm{MHz}, \mathrm{DMSO}-d_{6}\right)(\delta, \mathrm{ppm}): 8.04(\mathrm{~d}, 1 \mathrm{H}, J=6.8$ $\mathrm{Hz}, \mathrm{ArH}), 7.88-7.82(\mathrm{~m}, 4 \mathrm{H}, \mathrm{ArH}), 7.63(\mathrm{~d}, 3 \mathrm{H}, J=7.2 \mathrm{~Hz}$, ArH), $7.45(\mathrm{t}, 1 \mathrm{H}, J=7.2 \mathrm{~Hz}, \operatorname{ArH}), 7.05(\mathrm{~d}, 1 \mathrm{H}, J=6.8 \mathrm{~Hz}$, $\mathrm{ArH}), 5.94(\mathrm{~s}, 1 \mathrm{H}, \mathrm{CH}), 2.71(\mathrm{~d}, 1 \mathrm{H}, J=16.4 \mathrm{~Hz}, \mathrm{CH}), 2.43(\mathrm{~d}$, $1 \mathrm{H}, J=16.4 \mathrm{~Hz}, \mathrm{CH}), 2.30-2.22(\mathrm{~m}, 1 \mathrm{H}, \mathrm{CH}), 2.11-2.01(\mathrm{~m}, 1 \mathrm{H}$, $\mathrm{CH}), 1.13\left(\mathrm{~s}, 3 \mathrm{H}, \mathrm{CH}_{3}\right), 1.01\left(\mathrm{~s}, 3 \mathrm{H}, \mathrm{CH}_{3}\right), 0.92(\mathrm{t}, 3 \mathrm{H}, J=7.2$ $\mathrm{Hz}, \mathrm{CH}_{3}$ )

${ }^{13} \mathrm{C}$ NMR (100 MHz, DMSO- $\left.d_{6}\right)(\delta, \mathrm{ppm}): 192.8,173.1,142.6$, $140.7,136.1,133.5,131.7,131.4,129.6,129.0,128.7,128.0$, 127.9, 127.6, 127.0, 124.5, 123.7, 123.1, 120.1, 117.6, 69.5, 48.7, 26.9, 26.3, 24.7, 9.4;

IR $\left(\mathrm{KBr}, v, \mathrm{~cm}^{-1}\right): 2965,1740,1715,1667,1647,1540,1519$, 1490, 1459, 1382;

HRMS (ESI): $\mathrm{m} / \mathrm{z}$ calcd for: $\mathrm{C}_{29} \mathrm{H}_{24} \mathrm{BrNNaO}_{3}, \quad 536.0837$ $[\mathrm{M}+\mathrm{Na}]^{+}$; found: 536.0851 .

\section{9,9-Dimethyl-7-(4-nitrophenyl)-11-oxo-8,9,10,11-tetrahydro-} $7 \mathrm{H}$-acenaphtho[1,2-b]indol-8-yl propionate $(4 \mathrm{~h})$

A yellow solid: $0.331 \mathrm{~g}$, yield $69 \%$; Mp: $247-249^{\circ} \mathrm{C}$;

${ }^{1} \mathrm{H}$ NMR (400 MHz, DMSO- $d_{6}$ ) $(\delta, \mathrm{ppm}): 8.51(\mathrm{~d}, 2 \mathrm{H}, J=8.8$ $\mathrm{Hz}, \mathrm{ArH}), 8.07(\mathrm{~d}, 1 \mathrm{H}, J=6.4 \mathrm{~Hz}, \mathrm{ArH}), 8.00(\mathrm{~d}, 2 \mathrm{H}, J=8.8 \mathrm{~Hz}$, ArH), $7.85(\mathrm{~d}, 2 \mathrm{H}, J=8.0 \mathrm{~Hz}, \mathrm{ArH}), 7.67-7.63(\mathrm{~m}, 1 \mathrm{H}, \mathrm{ArH})$, 7.47-7.43 (m, 1H, ArH), $7.14(\mathrm{~d}, 1 \mathrm{H}, J=6.8 \mathrm{~Hz}, \mathrm{ArH}), 5.99$ (s, $1 \mathrm{H}, \mathrm{CH}), 2.73(\mathrm{~d}, 1 \mathrm{H}, J=16.8 \mathrm{~Hz}, \mathrm{CH}), 2.45(\mathrm{~d}, 1 \mathrm{H}, J=16.4$ $\mathrm{Hz}, \mathrm{CH}), 2.24-2.20(\mathrm{~m}, 1 \mathrm{H}, \mathrm{CH}), 2.10-2.02(\mathrm{~m}, 1 \mathrm{H}, \mathrm{CH}), 1.16$ (s, $\left.3 \mathrm{H}, \mathrm{CH}_{3}\right), 1.02\left(\mathrm{~s}, 3 \mathrm{H}, \mathrm{CH}_{3}\right), 0.87\left(\mathrm{t}, 3 \mathrm{H}, J=7.6 \mathrm{~Hz}, \mathrm{CH}_{3}\right)$;

${ }^{13} \mathrm{C}$ NMR (100 MHz, DMSO- $\left.d_{6}\right)(\delta, \mathrm{ppm}): 192.9,173.1,148.1$, $142.6,142.3,140.5,131.7,131.2,129.6,128.7,128.4,127.9$, 127.8(0), 127.7(7), 127.2, 125.9, 125.1, 124.0, 120.5, 118.1, 69.5, 48.6, 26.9, 26.2, 24.8, 9.4;

IR $\left(\mathrm{KBr}, v, \mathrm{~cm}^{-1}\right): 2978,1740,1666,1609,1594,1522,1497$, 1457, 1385, 1342;

HRMS (ESI): m/z calcd for: $\mathrm{C}_{29} \mathrm{H}_{24} \mathrm{~N}_{2} \mathrm{NaO}_{5}, 503.1583$ [M+Na] $]^{+}$; found: 503.1597 .

9,9-Dimethyl-11-oxo-7-phenyl-8,9,10,11-tetrahydro-7Hacenaphtho[1,2-b]indol-8-yl propionate (4i) A yellow solid: $0.313 \mathrm{~g}$, yield $72 \%$; Mp: $182-184{ }^{\circ} \mathrm{C}$; ${ }^{1} \mathrm{H}$ NMR $\left(400 \mathrm{MHz}\right.$, DMSO- $\left.d_{6}\right)(\delta, \mathrm{ppm}): 8.04(\mathrm{~d}, 1 \mathrm{H}, J=6.4$ $\mathrm{Hz}, \mathrm{ArH}), 7.81$ (d, 2H, J = 5.6 Hz, ArH), 7.65 (s, 6H, ArH), 7.43 $(\mathrm{t}, 1 \mathrm{H}, J=7.2 \mathrm{~Hz}, \mathrm{ArH}), 6.97(\mathrm{~d}, 1 \mathrm{H}, J=6.8 \mathrm{~Hz}, \mathrm{ArH}), 5.95(\mathrm{~s}$, $1 \mathrm{H}, \mathrm{CH}), 2.71(\mathrm{~d}, 1 \mathrm{H}, J=16.8 \mathrm{~Hz}, \mathrm{CH}), 2.43(\mathrm{~d}, 1 \mathrm{H}, J=16.8$ 
$\mathrm{Hz}, \mathrm{CH}), 2.24-2.18(\mathrm{~m}, 1 \mathrm{H}, \mathrm{CH}), 2.08-2.00$ (m, 1H, CH), 1.14 (s, $\left.3 \mathrm{H}, \mathrm{CH}_{3}\right), 1.00\left(\mathrm{~s}, 3 \mathrm{H}, \mathrm{CH}_{3}\right), 0.89\left(\mathrm{t}, 3 \mathrm{H}, J=7.6 \mathrm{~Hz}, \mathrm{CH}_{3}\right)$; ${ }^{13} \mathrm{C}$ NMR $\left(100 \mathrm{MHz}, \mathrm{DMSO}-d_{6}\right)(\delta, \mathrm{ppm}): 192.8,172.9,142.5$, $140.8,136.8,131.8,131.5,130.5,130.0,129.6,128.7,128.2$, $127.8,127.4,126.9,126.8,124.4,123.6,119.9,117.4,69.5,48.7$, $26.9,26.3,24.8,9.4$;

IR $\left(\mathrm{KBr}, v, \mathrm{~cm}^{-1}\right): 3067,1738,1661,1611,1596,1518,1457$, 1411, 1392, 1350;

HRMS (ESI): $\mathrm{m} / \mathrm{z}$ calcd for: $\mathrm{C}_{29} \mathrm{H}_{25} \mathrm{NNaO}_{3}, 458.1732[\mathrm{M}+\mathrm{Na}]^{+}$; found: 458.1749 .

\section{9,9-Dimethyl-11-oxo-7-(m-tolyl)-8,9,10,11-tetrahydro-7H- acenaphtho[1,2-b]indol-8-yl propionate $(4 \mathbf{j})$}

A yellow solid: $0.314 \mathrm{~g}$, yield $70 \%$; Mp: $156-158^{\circ} \mathrm{C}$;

${ }^{1} \mathrm{H}$ NMR $\left(400 \mathrm{MHz}, \mathrm{DMSO}-d_{6}\right)(\delta, \mathrm{ppm}): 8.04(\mathrm{~d}, 1 \mathrm{H}, J=6.4$ $\mathrm{Hz}, \mathrm{ArH}), 7.83-7.80(\mathrm{~m}, 2 \mathrm{H}, \mathrm{ArH}), 7.63(\mathrm{t}, 1 \mathrm{H}, J=7.2 \mathrm{~Hz}, \mathrm{ArH})$, $7.55(\mathrm{t}, 1 \mathrm{H}, J=8.0 \mathrm{~Hz}, \operatorname{ArH}), 7.46-7.39(\mathrm{~m}, 4 \mathrm{H}, \operatorname{ArH}), 7.01(\mathrm{~d}$, $1 \mathrm{H}, J=7.2 \mathrm{~Hz}, \mathrm{ArH}), 5.93(\mathrm{~s}, 1 \mathrm{H}, \mathrm{CH}), 2.71(\mathrm{~d}, 1 \mathrm{H}, J=16.0 \mathrm{~Hz}$, $\mathrm{CH}), 2.47(\mathrm{~d}, 1 \mathrm{H}, J=12.4 \mathrm{~Hz}, \mathrm{CH}), 2.42\left(\mathrm{~s}, 3 \mathrm{H}, \mathrm{CH}_{3}\right), 2.26-2.12$ $(\mathrm{m}, 2 \mathrm{H}, \mathrm{CH}), 1.11\left(\mathrm{~s}, 3 \mathrm{H}, \mathrm{CH}_{3}\right), 1.00\left(\mathrm{~s}, 3 \mathrm{H}, \mathrm{CH}_{3}\right), 0.92(\mathrm{t}, 3 \mathrm{H}, J$ $\left.=6.0 \mathrm{~Hz}, \mathrm{CH}_{3}\right)$;

${ }^{13} \mathrm{C}$ NMR $\left(100 \mathrm{MHz}, \mathrm{DMSO}-d_{6}\right)(\delta, \mathrm{ppm}): 193.7,175.6,147.9$, $140.1,139.4,137.1,131.9,131.8,130.2,130.1,129.5,128.8$, $128.6,127.8,127.1,126.7,126.6,124.8,123.4,123.3,119.8$, $115.9,68.3,47.3,27.3,26.3,25.9,21.4,9.5$;

IR $\left(\mathrm{KBr}, v, \mathrm{~cm}^{-1}\right): 3059,1742,1666,1647,1610,1568,1540$, 1521, 1489, 1471, 1385;

HRMS (ESI): m/z calcd for: $\mathrm{C}_{30} \mathrm{H}_{27} \mathrm{NNaO}_{3}, 472.1889[\mathrm{M}+\mathrm{Na}]^{+}$; found: 472.1898 .

\section{9,9-Dimethyl-11-oxo-7-(o-tolyl)-8,9,10,11-tetrahydro-7H- acenaphtho[1,2-b]indol-8-yl propionate (4k) \\ A yellow solid: $0.287 \mathrm{~g}$, yield $64 \%$; Mp: $190-192{ }^{\circ} \mathrm{C}$;}

${ }^{1} \mathrm{H}$ NMR $\left(400 \mathrm{MHz}, \mathrm{DMSO}-d_{6}\right)(\delta, \mathrm{ppm}): 8.03(\mathrm{~d}, 1 \mathrm{H}, J=6.8$ $\mathrm{Hz}, \operatorname{ArH}), 7.83-7.79(\mathrm{~m}, 2 \mathrm{H}, \operatorname{ArH}), 7.66-7.62(\mathrm{~m}, 1 \mathrm{H}, \operatorname{ArH})$, 7.58-7.55 (m, 2H, ArH), 7.49-7.48 (m, 1H, ArH), 7.44-7.38 (m, $2 \mathrm{H}, \mathrm{ArH}), 6.69(\mathrm{~d}, 1 \mathrm{H}, J=7.2 \mathrm{~Hz}, \mathrm{ArH}), 5.92+5.60(\mathrm{~s}, 1 \mathrm{H}$, $\mathrm{CH}), 2.78-2.66(\mathrm{~m}, 1 \mathrm{H}, \mathrm{CH}), 2.46-2.32(\mathrm{~m}, 1 \mathrm{H}, \mathrm{CH}), 2.30-2.20$ $(\mathrm{m}, 1 \mathrm{H}, \mathrm{CH}), 2.16-2.12+1.94-1.88(\mathrm{~m}, 1 \mathrm{H}, \mathrm{CH}), 1.13(\mathrm{~d}, 3 \mathrm{H}, J$ $\left.=4.4 \mathrm{~Hz}, \mathrm{CH}_{3}\right), 1.00\left(\mathrm{~d}, 3 \mathrm{H}, J=8.0 \mathrm{~Hz}, \mathrm{CH}_{3}\right), 0.95+0.85(\mathrm{t}, 3 \mathrm{H}$, $\left.J=7.6 \mathrm{~Hz}, \mathrm{CH}_{3}\right)$;

${ }^{13} \mathrm{C}$ NMR (100 MHz, DMSO- $\left.d_{6}\right)(\delta, \mathrm{ppm}): 192.7(3), 192.6(6)$, $172.9,172.6,142.6,142.5,140.8,140.7,135.9,135.5,135.4$, 135.2, 131.9, 131.7(63), 131.7(57), 131.7(3), 131.7(0), 131.6(4), $130.5(9), 130.4,129.6,129.5,128.7,128.3,128.2,128.0(0)$, 127.9(8), 127.9(5), 127.9(3), 127.8, 127.4(3), 127.4(1), 126.8(4), 126.8(2), 124.1(1), 124.0(6), 123.6, 119.3, 119.1, 117.1, 117.0, $69.5,69.3,49.0,48.4,27.0,26.6,26.3,26.0,24.9,24.3,17.2,9.4$, 9.3 ;

IR $\left(\mathrm{KBr}, v, \mathrm{~cm}^{-1}\right): 3070,1755,1693,1604,1581,1553,1476$, 1386, 1357, 1258;

HRMS (ESI): $\mathrm{m} / \mathrm{z}$ calcd for: $\mathrm{C}_{30} \mathrm{H}_{27} \mathrm{NNaO}_{3}, 472.1889[\mathrm{M}+\mathrm{Na}]^{+}$; found: 472.1903 .

\section{7-(4-Methoxyphenyl)-9,9-dimethyl-11-oxo-8,9,10,11-} tetrahydro-7 $H$-acenaphtho[1,2-b]indol-8-yl propionate (4I) A yellow solid: $0.358 \mathrm{~g}$, yield $77 \%$; Mp: $194-196{ }^{\circ} \mathrm{C}$;

${ }^{1} \mathrm{H}$ NMR $\left(400 \mathrm{MHz}, \mathrm{DMSO}-d_{6}\right)(\delta, \mathrm{ppm}): 8.02(\mathrm{~d}, 1 \mathrm{H}, J=6.4$ $\mathrm{Hz}, \operatorname{ArH}), 7.81(\mathrm{~d}, 2 \mathrm{H}, J=7.6 \mathrm{~Hz}, \operatorname{ArH}), 7.63(\mathrm{t}, 1 \mathrm{H}, J=7.2 \mathrm{~Hz}$, $\mathrm{ArH}), 7.53$ (s, 2H, ArH), $7.43(\mathrm{t}, 1 \mathrm{H}, J=7.2 \mathrm{~Hz}, \mathrm{ArH}), 7.18(\mathrm{~d}$, $2 \mathrm{H}, J=7.6 \mathrm{~Hz}, \mathrm{ArH}), 6.98(\mathrm{~d}, 1 \mathrm{H}, J=6.8 \mathrm{~Hz}, \mathrm{ArH}), 5.88(\mathrm{~s}, 1 \mathrm{H}$, $\mathrm{CH}), 3.89\left(\mathrm{~s}, 3 \mathrm{H}, \mathrm{OCH}_{3}\right), 2.72(\mathrm{~d}, 1 \mathrm{H}, J=16.4 \mathrm{~Hz}, \mathrm{CH}), 2.39(\mathrm{~d}$, $1 \mathrm{H}, J=16.4 \mathrm{~Hz}, \mathrm{CH}), 2.31-2.21(\mathrm{~m}, 1 \mathrm{H}, \mathrm{CH}), 2.16-2.06(\mathrm{~m}, 1 \mathrm{H}$, $\mathrm{CH}), 1.13\left(\mathrm{~s}, 3 \mathrm{H}, \mathrm{CH}_{3}\right), 1.00\left(\mathrm{~s}, 3 \mathrm{H}, \mathrm{CH}_{3}\right), 0.93(\mathrm{t}, 3 \mathrm{H}, J=7.2$ $\left.\mathrm{Hz}, \mathrm{CH}_{3}\right)$;
${ }^{13} \mathrm{C}$ NMR $\left(100 \mathrm{MHz}, \mathrm{DMSO}-d_{6}\right)(\delta, \mathrm{ppm}): 192.8,173.0,160.2$, $142.8,141.1,131.8,131.6,129.6,129.3,128.7,128.3,128.1$, $127.9,127.4,126.8,124.1,123.5,119.9,117.2,115.5,69.4,56.1$, 48.6, 27.0, 26.2, 24.9, 9.4;

IR $\left(\mathrm{KBr}, v, \mathrm{~cm}^{-1}\right): 3063,1741,1660,1609,1515,1462,1410$, 1297, 1252, 1171;

HRMS (ESI): $\mathrm{m} / \mathrm{z}$ calcd for: $\mathrm{C}_{30} \mathrm{H}_{27} \mathrm{NNaO}_{4}, 488.1838[\mathrm{M}+\mathrm{Na}]^{+}$; found: 488.1849 .

7-(4-Bromobenzyl)-9,9-dimethyl-11-oxo-8,9,10,11-tetrahydro$\mathbf{7 H}$-acenaphtho[1,2-b]indol-8-yl propionate $(\mathbf{4 m})$

A yellow solid: $0.374 \mathrm{~g}$, yield $71 \%$; Mp: $150-152{ }^{\circ} \mathrm{C}$;

${ }^{1} \mathrm{H}$ NMR (400 MHz, DMSO- $\left.d_{6}\right)(\delta, \mathrm{ppm}): 8.02(\mathrm{~d}, 1 \mathrm{H}, J=6.8$ $\mathrm{Hz}, \mathrm{ArH}), 7.80(\mathrm{~d}, 2 \mathrm{H}, J=8.4 \mathrm{~Hz}, \mathrm{ArH}), 7.64-7.58(\mathrm{~m}, 2 \mathrm{H}$, ArH), $7.53(\mathrm{~d}, 2 \mathrm{H}, J=8.0 \mathrm{~Hz}, \mathrm{ArH}), 7.48(\mathrm{t}, 1 \mathrm{H}, J=7.6 \mathrm{~Hz}$, $\mathrm{ArH}), 7.02(\mathrm{~d}, 2 \mathrm{H}, J=8.0 \mathrm{~Hz}, \mathrm{ArH}), 6.04(\mathrm{~s}, 1 \mathrm{H}, \mathrm{CH}), 5.62(\mathrm{~s}$, $\left.2 \mathrm{H}, \mathrm{CH}_{2}\right), 2.80(\mathrm{~d}, 1 \mathrm{H}, J=16.8 \mathrm{~Hz}, \mathrm{CH}), 2.27(\mathrm{~d}, 1 \mathrm{H}, J=16.4$ $\mathrm{Hz}, \mathrm{CH}), 2.23-2.17(\mathrm{~m}, 1 \mathrm{H}, \mathrm{CH}), 2.01-1.91(\mathrm{~m}, 1 \mathrm{H}, \mathrm{CH}), 1.08$ (s, $\left.3 \mathrm{H}, \mathrm{CH}_{3}\right), 1.05\left(\mathrm{~s}, 3 \mathrm{H}, \mathrm{CH}_{3}\right), 0.85\left(\mathrm{t}, 3 \mathrm{H}, J=7.6 \mathrm{~Hz}, \mathrm{CH}_{3}\right)$;

${ }^{13} \mathrm{C}$ NMR (100 MHz, DMSO- $\left.d_{6}\right)(\delta, \mathrm{ppm}): 192.7,173.6,142.5$, $140.3,136.4,132.1,131.8(3), 131.7(5), 129.5,128.6,128.4$, $128.2,127.9,127.3,126.8,124.8,123.4,121.0,120.7,116.9$, 68.7, 48.9, 47.6, 26.9, 25.9, 25.7, 9.1;

IR $\left(\mathrm{KBr}, v, \mathrm{~cm}^{-1}\right): 3065,1728,1681,1646,1532,1498,1456$, $1437,1388,1352$

HRMS (ESI): $\mathrm{m} / \mathrm{z}$ calcd for: $\mathrm{C}_{30} \mathrm{H}_{26} \mathrm{BrNNaO}_{3}, \quad 550.0994$ $[\mathrm{M}+\mathrm{Na}]^{+}$; found: 550.0978 .

\section{9,9-Dimethyl-11-oxo-7-phenyl-8,9,10,11-tetrahydro-7H-} acenaphtho[1,2-b]indol-8-yl butyrate (4n) A yellow solid: $0.323 \mathrm{~g}$, yield $72 \%$; Mp: $196-198{ }^{\circ} \mathrm{C}$;

${ }^{1} \mathrm{H}$ NMR (400 MHz, DMSO- $d_{6}$ ) $(\delta, \mathrm{ppm}): 8.05(\mathrm{~d}, 1 \mathrm{H}, J=5.6$ $\mathrm{Hz}, \mathrm{ArH}), 7.80$ (s, 2H, ArH), 7.65 (s, 6H, ArH), 7.41 (t, 1H, $J=$ $6.8 \mathrm{~Hz}, \mathrm{ArH}), 6.96(\mathrm{~d}, 1 \mathrm{H}, J=6.4 \mathrm{~Hz}, \mathrm{ArH}), 5.95(\mathrm{~s}, 1 \mathrm{H}, \mathrm{CH})$, $2.72(\mathrm{~d}, 1 \mathrm{H}, J=16.4 \mathrm{~Hz}, \mathrm{CH}), 2.41(\mathrm{~d}, 1 \mathrm{H}, J=16.4 \mathrm{~Hz}, \mathrm{CH})$, 2.18-2.14 (m, 1H, CH), 2.02-1.98 (m, 1H, CH), 1.40-1.39 (m, $\left.2 \mathrm{H}, \mathrm{CH}_{2}\right), 1.14\left(\mathrm{~s}, 3 \mathrm{H}, \mathrm{CH}_{3}\right), 1.00\left(\mathrm{~s}, 3 \mathrm{H}, \mathrm{CH}_{3}\right), 0.79(\mathrm{t}, 3 \mathrm{H}, J=$ $\left.6.4 \mathrm{~Hz}, \mathrm{CH}_{3}\right)$;

${ }^{13} \mathrm{C}$ NMR (100 MHz, DMSO- $\left.d_{6}\right)(\delta, \mathrm{ppm}): 192.8,172.0,142.5$, $140.8,136.7,131.8,131.5,130.6,130.0,129.6,128.7,128.2$, 127.8, 127.4, 126.9, 126.7, 124.4, 123.7, 119.9, 117.4, 69.4, 48.6, $35.3,26.2,24.9,18.2,13.9$;

IR $\left(\mathrm{KBr}, v, \mathrm{~cm}^{-1}\right)$ : 3056, 1755, 1693, 1615, 1523, 1488, 1473, $1465,1385,1301$;

HRMS (ESI): $\mathrm{m} / \mathrm{z}$ calcd for: $\mathrm{C}_{30} \mathrm{H}_{27} \mathrm{NNaO}_{3}, 472.1889[\mathrm{M}+\mathrm{Na}]^{+}$; found: 472.1898 .

\section{7-(4-Methoxyphenyl)-9,9-dimethyl-11-oxo-8,9,10,11-} tetrahydro-7H-acenaphtho[1,2-b]indol-8-yl isobutyrate (4o) A yellow solid: $0.326 \mathrm{~g}$, yield $68 \%$; Mp: $140-142{ }^{\circ} \mathrm{C}$;

${ }^{1} \mathrm{H}$ NMR (400 MHz, DMSO- $\left.d_{6}\right)(\delta, \mathrm{ppm}): 8.03(\mathrm{~d}, 1 \mathrm{H}, J=6.8$ $\mathrm{Hz}, \mathrm{ArH}), 7.83-7.80(\mathrm{~m}, 2 \mathrm{H}, \mathrm{ArH}), 7.63(\mathrm{t}, 1 \mathrm{H}, J=7.2 \mathrm{~Hz}, \mathrm{ArH})$, $7.54(\mathrm{~s}, 2 \mathrm{H}, \mathrm{ArH}), 7.44(\mathrm{t}, 1 \mathrm{H}, J=7.2 \mathrm{~Hz}, \mathrm{ArH}), 7.18(\mathrm{~d}, 2 \mathrm{H}, J=$ $8.4 \mathrm{~Hz}, \mathrm{ArH}), 6.99(\mathrm{~d}, 1 \mathrm{H}, J=6.8 \mathrm{~Hz}, \mathrm{ArH}), 5.90(\mathrm{~s}, 1 \mathrm{H}, \mathrm{CH})$, $3.89\left(\mathrm{~s}, 3 \mathrm{H}, \mathrm{OCH}_{3}\right), 2.74(\mathrm{~d}, 1 \mathrm{H}, J=16.4 \mathrm{~Hz}, \mathrm{CH}), 2.41-2.40(\mathrm{~m}$, $1 \mathrm{H}, \mathrm{CH}), 2.38(\mathrm{~d}, 1 \mathrm{H}, J=6.0 \mathrm{~Hz}, \mathrm{CH}), 1.14\left(\mathrm{~s}, 3 \mathrm{H}, \mathrm{CH}_{3}\right), 1.04$ $\left(\mathrm{d}, 3 \mathrm{H}, J=7.2 \mathrm{~Hz}, \mathrm{CH}_{3}\right), 1.00\left(\mathrm{~s}, 3 \mathrm{H}, \mathrm{CH}_{3}\right), 0.94(\mathrm{~d}, 3 \mathrm{H}, J=6.8$ $\left.\mathrm{Hz}, \mathrm{CH}_{3}\right)$;

${ }^{13} \mathrm{C}$ NMR (100 MHz, DMSO- $\left.d_{6}\right)(\delta, \mathrm{ppm}): 192.8,175.3,160.2$, $142.7,141.1,131.8,131.6,129.6,129.3,128.7,128.3,128.1$, $127.9,127.4,126.9,124.1,123.6,119.9,117.2,115.6,69.2,56.1$, $48.5,33.7,26.2,24.9,19.5,18.9$;

IR $\left(\mathrm{KBr}, v, \mathrm{~cm}^{-1}\right): 3056,1762,1664,1658,1568,1489,1473$, 1465, 1386, 1355;

HRMS (ESI): $\mathrm{m} / \mathrm{z}$ calcd for: $\mathrm{C}_{31} \mathrm{H}_{29} \mathrm{NNaO}_{4}, 502.1994[\mathrm{M}+\mathrm{Na}]^{+}$; found: 502.2008 . 
General Procedure for the Synthesis of 6a-6f

7-(4-Chlorophenyl)-10,10-dimethyl-7H-acenaphtho[1,2b]indol-11(10H)-one (6a)

In a typical experiment procedure, 3-((4-chlorophenyl)amino)6,6-dimethylcyclohex-2-enone $5 \mathbf{a}(1.0 \mathrm{mmol}, 0.249 \mathrm{~g}, 1.0$ equiv) and acenaphthylene-1,2-dione 2 (1.0 mmol, $0.182 \mathrm{~g}, 1.0$ equiv) were introduced in a $10-\mathrm{mL}$ Initiator ${ }^{\mathrm{TM}}$ reaction vial, acetic anhydride $(1.5 \mathrm{~mL})$ was then successively added. Subsequently, the reaction vial was capped and then pre-stirring for 20 second. The mixture was irradiated (Time: $23 \mathrm{~min}$, Temperature: $120{ }^{\circ} \mathrm{C}$; Absorption Level: High; Fixed Hold Time) until TLC revealed that conversion of the starting materials were completed. The reaction mixture was then cooled to room temperature and then diluted with cold water $(25 \mathrm{~mL})$. The solid product was collected by Büchner filtration to give pure aromatized indoles $6 \mathbf{6}$, which was further purified by washing from $95 \%$ ethanol.

An orange solid: $0.304 \mathrm{~g}$, yield $77 \%$; Mp: $266-268^{\circ} \mathrm{C}$;

${ }^{1} \mathrm{H}$ NMR (400 MHz, DMSO- $\left.d_{6}\right)(\delta, \mathrm{ppm}): 8.10(\mathrm{~d}, 1 \mathrm{H}, J=6.0$ $\mathrm{Hz}, \mathrm{ArH}), 7.82-7.81(\mathrm{~m}, 6 \mathrm{H}, \mathrm{ArH}), 7.64(\mathrm{t}, 1 \mathrm{H}, J=6.8 \mathrm{~Hz}, \mathrm{ArH})$, $7.47(\mathrm{t}, 1 \mathrm{H}, J=6.0 \mathrm{~Hz}, \mathrm{ArH}), 7.22(\mathrm{~d}, 1 \mathrm{H}, J=6.0 \mathrm{~Hz}, \mathrm{ArH}), 6.51$ $(\mathrm{d}, 1 \mathrm{H}, J=9.6 \mathrm{~Hz}, \mathrm{CH}), 6.28(\mathrm{~d}, 1 \mathrm{H}, J=9.6 \mathrm{~Hz}, \mathrm{CH}), 1.28$ (s, $\left.6 \mathrm{H}, \mathrm{CH}_{3}\right)$;

${ }^{13} \mathrm{C}$ NMR (100 MHz, DMSO- $\left.d_{6}\right)(\delta, \mathrm{ppm}): 198.2,144.7,144.3$, $139.9,135.3,134.2,132.0,131.4,130.7,129.5,128.6,128.4$, $128.2,128.0,127.3,127.2,126.0,123.9,120.1,114.2,112.3$, 47.0, 25.7;

IR $\left(\mathrm{KBr}, v, \mathrm{~cm}^{-1}\right): 3059,1645,1609,1591,1518,1492,1476$, 1454, 1433, 1417, 1385, 1374;

HRMS (ESI): m/z calcd for: $\mathrm{C}_{26} \mathrm{H}_{19} \mathrm{ClNO}, 396.1155[\mathrm{M}+\mathrm{H}]^{+}$; found: 396.1167.

\section{7-(3,4-Dichlorophenyl)-10,10-dimethyl-7H-acenaphtho[1,2- $b$ ]indol-11(10H)-one $(6 \mathrm{~b})$}

An orange solid: $0.292 \mathrm{~g}$, yield $68 \%$; Mp: $205-207^{\circ} \mathrm{C}$;

${ }^{1} \mathrm{H}$ NMR (400 MHz, DMSO- $\left.d_{6}\right)(\delta, \mathrm{ppm}): 8.20(\mathrm{~d}, 1 \mathrm{H}, J=2.4$ $\mathrm{Hz}, \mathrm{ArH}), 8.10(\mathrm{~d}, 1 \mathrm{H}, J=6.8 \mathrm{~Hz}, \mathrm{ArH}), 8.00(\mathrm{~d}, 1 \mathrm{H}, J=8.8 \mathrm{~Hz}$, ArH), 7.86-7.82 (m, 3H, ArH), $7.64(\mathrm{t}, 1 \mathrm{H}, J=7.2 \mathrm{~Hz}, \mathrm{ArH})$, 7.49 (t, $1 \mathrm{H}, J=7.2 \mathrm{~Hz}, \operatorname{ArH}), 7.25(\mathrm{~d}, 1 \mathrm{H}, J=7.2 \mathrm{~Hz}, \mathrm{ArH}), 6.56$ $(\mathrm{d}, 1 \mathrm{H}, J=10.0 \mathrm{~Hz}, \mathrm{CH}), 6.30(\mathrm{~d}, 1 \mathrm{H}, J=9.6 \mathrm{~Hz}, \mathrm{CH}), 1.28$ (s, $\left.6 \mathrm{H}, \mathrm{CH}_{3}\right)$;

${ }^{13} \mathrm{C}$ NMR (100 MHz, DMSO- $\left.d_{6}\right)(\delta, \mathrm{ppm}): 198.2,144.8,144.5$, $139.9,136.3,133.0,132.6,132.5,132.0,131.3,129.5,128.8$, $128.6,128.1,128.0,127.4,127.3,127.1,126.1,124.0,120.1$, 114.3, 112.3, 47.1, 25.7;

IR $\left(\mathrm{KBr}, v, \mathrm{~cm}^{-1}\right): 3061,1738,1669,1611,1519,1481,1460$, 1434, 1411, 1390, 1349;

HRMS (ESI): m/z calcd for: $\mathrm{C}_{26} \mathrm{H}_{18} \mathrm{Cl}_{2} \mathrm{NO}, 430.0765[\mathrm{M}+\mathrm{H}]^{+}$; found: 430.0749 .

\section{7-(4-Bromophenyl)-10,10-dimethyl-7H-acenaphtho[1,2- $b$ ]indol-11(10H)-one (6c)}

An orange solid: $0.329 \mathrm{~g}$, yield $75 \%$; Mp: $267-269^{\circ} \mathrm{C}$;

${ }^{1} \mathrm{H}$ NMR (400 MHz, DMSO- $\left.d_{6}\right)(\delta, \mathrm{ppm}): 8.10(\mathrm{~d}, 1 \mathrm{H}, J=6.8$ $\mathrm{Hz}, \mathrm{ArH}), 7.93(\mathrm{~d}, 2 \mathrm{H}, J=8.0 \mathrm{~Hz}, \mathrm{ArH}), 7.82(\mathrm{t}, 2 \mathrm{H}, J=7.2 \mathrm{~Hz}$, ArH), $7.76(\mathrm{~d}, 2 \mathrm{H}, J=8.0 \mathrm{~Hz}, \mathrm{ArH}), 7.64(\mathrm{~d}, 1 \mathrm{H}, J=7.2 \mathrm{~Hz}$, ArH), $7.47(\mathrm{~d}, 1 \mathrm{H}, J=7.2 \mathrm{~Hz}, \mathrm{ArH}), 7.23(\mathrm{~d}, 1 \mathrm{H}, J=7.2 \mathrm{~Hz}$, ArH), $6.52(\mathrm{~d}, 1 \mathrm{H}, J=10.0 \mathrm{~Hz}, \mathrm{CH}), 6.28(\mathrm{~d}, 1 \mathrm{H}, J=10.0 \mathrm{~Hz}$, $\mathrm{CH}), 1.28\left(\mathrm{~s}, 6 \mathrm{H}, \mathrm{CH}_{3}\right)$;

${ }^{13} \mathrm{C}$ NMR (100 MHz, DMSO- $\left.d_{6}\right)(\delta, \mathrm{ppm}): 198.2,144.7,144.2$, $139.8,135.7,133.7,132.0,131.3,129.5,128.6(5), 128.6(0)$, $128.2,128.0,127.3,127.2,126.1,123.9,122.6,120.1,114.2$, 112.3, 47.0, 25.7;

IR $\left(\mathrm{KBr}, \mathrm{v}, \mathrm{cm}^{-1}\right): 3055,1646,1609,1584,1517,1490,1454$, 1433, 1417, 1385, 1349;

HRMS (ESI): $\mathrm{m} / \mathrm{z}$ calcd for: $\mathrm{C}_{26} \mathrm{H}_{19} \mathrm{BrNO}, 440.0650[\mathrm{M}+\mathrm{H}]^{+}$; found: 440.0639 .
10,10-Dimethyl-7-phenyl-7H-acenaphtho[1,2-b]indol11(10H)-one (6d)

An orange solid: $0.267 \mathrm{~g}$, yield $74 \%$; Mp: $176-178^{\circ} \mathrm{C}$;

${ }^{1} \mathrm{H}$ NMR (400 MHz, DMSO- $\left.d_{6}\right)(\delta, \mathrm{ppm}): 8.10$ (d, $1 \mathrm{H}, J=6.8$ $\mathrm{Hz}, \mathrm{ArH}), 7.84-7.73$ (m, 6H, ArH), 7.69-7.62 (m, 2H, ArH), 7.47 $(\mathrm{t}, 1 \mathrm{H}, J=7.2 \mathrm{~Hz}, \mathrm{ArH}), 7.18(\mathrm{~d}, 1 \mathrm{H}, J=6.8 \mathrm{~Hz}, \operatorname{ArH}), 6.51(\mathrm{~d}$, $1 \mathrm{H}, J=10.0 \mathrm{~Hz}, \mathrm{CH}), 6.28(\mathrm{~d}, 1 \mathrm{H}, J=9.6 \mathrm{~Hz}, \mathrm{CH}), 1.28(\mathrm{~s}, 6 \mathrm{H}$, $\mathrm{CH}_{3}$;

${ }^{13} \mathrm{C}$ NMR (100 MHz, DMSO- $\left.d_{6}\right)(\delta, \mathrm{ppm}): 198.2,144.6,144.2$, $140.0,136.4,132.1,131.5,130.7,129.7,129.5,128.6,128.4$, $127.9,127.3,127.1,126.5,125.9,123.8,119.9,114.1,112.4$, 47.0, 25.7;

IR $\left(\mathrm{KBr}, v, \mathrm{~cm}^{-1}\right): 3043,1654,1639,1580,1523,1467,1429$, 1417, 1387, 1366;

HRMS (ESI): $\mathrm{m} / \mathrm{z}$ calcd for: $\mathrm{C}_{26} \mathrm{H}_{20} \mathrm{NO}, 362.1545[\mathrm{M}+\mathrm{H}]^{+}$; found: 362.1561 .

\section{0,10-Dimethyl-7-(p-tolyl)-7H-acenaphtho[1,2-b]indol-} 11(10H)-one $(6 \mathrm{e})$

An orange solid: $0.319 \mathrm{~g}$, yield $85 \%$; Mp: $238-240{ }^{\circ} \mathrm{C}$;

${ }^{1} \mathrm{H}$ NMR (400 MHz, DMSO- $\left.d_{6}\right)(\delta, \mathrm{ppm}): 8.08(\mathrm{~d}, 1 \mathrm{H}, J=6.0$ $\mathrm{Hz}, \mathrm{ArH}), 7.81$ (t, 2H, $J=7.2 \mathrm{~Hz}, \operatorname{ArH}), 7.63(\mathrm{~d}, 3 \mathrm{H}, J=7.2 \mathrm{~Hz}$, $\operatorname{ArH}), 7.52(\mathrm{~d}, 2 \mathrm{H}, J=6.4 \mathrm{~Hz}, \mathrm{ArH}), 7.45(\mathrm{t}, 1 \mathrm{H}, J=6.8 \mathrm{~Hz}$, $\operatorname{ArH}), 7.17(\mathrm{~d}, 1 \mathrm{H}, J=6.4 \mathrm{~Hz}, \operatorname{ArH}), 6.48(\mathrm{~d}, 1 \mathrm{H}, J=9.6 \mathrm{~Hz}$, $\mathrm{CH}), 6.25$ (d, $1 \mathrm{H}, J=9.2 \mathrm{~Hz}, \mathrm{CH}), 2.48\left(\mathrm{~s}, 3 \mathrm{H}, \mathrm{CH}_{3}\right), 1.27$ (s, 6H, $\mathrm{CH}_{3}$;

${ }^{13} \mathrm{C}$ NMR (100 MHz, DMSO- $\left.d_{6}\right)(\delta, \mathrm{ppm}): 198.1,144.4,144.2$, $140.1,139.3,133.8,132.1,131.5,131.1,129.5,128.6,128.4$, $127.9,127.2,127.0,126.2,125.8,123.8,119.9,114.0,112.4$, 47.0, 25.7, 21.3;

IR $\left(\mathrm{KBr}, v, \mathrm{~cm}^{-1}\right): 3063,1736,1654,1639,1568,1543,1485$, 1476, 1415, 1386;

HRMS (ESI): $\mathrm{m} / \mathrm{z}$ calcd for: $\mathrm{C}_{27} \mathrm{H}_{22} \mathrm{NO}, 376.1701[\mathrm{M}+\mathrm{H}]^{+}$; found: 376.1718 .

\section{7-(4-Methoxyphenyl)-10,10-dimethyl-7H-acenaphtho[1,2-} b]indol-11(10H)-one (6f)

An orange solid: $0.313 \mathrm{~g}$, yield $80 \%$; Mp: $240-242{ }^{\circ} \mathrm{C}$;

${ }^{1} \mathrm{H}$ NMR (400 MHz, DMSO- $d_{6}$ ) $(\delta, \mathrm{ppm}): 8.08(\mathrm{~d}, 1 \mathrm{H}, J=6.8$ $\mathrm{Hz}, \mathrm{ArH}), 7.80$ (t, $2 \mathrm{H}, J=8.0 \mathrm{~Hz}, \operatorname{ArH}), 7.68(\mathrm{~d}, 2 \mathrm{H}, J=8.8 \mathrm{~Hz}$, ArH), 7.65-7.61 (m, 1H, ArH), $7.45(\mathrm{t}, 1 \mathrm{H}, J=7.2 \mathrm{~Hz}, \mathrm{ArH})$, $7.25(\mathrm{~d}, 2 \mathrm{H}, J=8.8 \mathrm{~Hz}, \operatorname{ArH}), 7.16(\mathrm{~d}, 1 \mathrm{H}, J=6.8 \mathrm{~Hz}, \operatorname{ArH})$, $6.46(\mathrm{~d}, 1 \mathrm{H}, J=10.0 \mathrm{~Hz}, \mathrm{CH}), 6.25(\mathrm{~d}, 1 \mathrm{H}, J=9.6 \mathrm{~Hz}, \mathrm{CH}), 3.91$ (s, $\left.3 \mathrm{H}, \mathrm{OCH}_{3}\right), 1.27\left(\mathrm{~s}, 6 \mathrm{H}, \mathrm{CH}_{3}\right)$;

${ }^{13} \mathrm{C}$ NMR (100 MHz, DMSO- $\left.d_{6}\right)(\delta, \mathrm{ppm}): 198.1,160.0,144.4$, $144.3,140.4,132.1,131.6,129.5,129.0,128.6,128.4,127.9$, $127.8,127.2$, 127.0, 125.6, 123.7, 119.9, 115.7, 113.9, 112.4, 56.1, 46.9, 25.7;

IR $\left(\mathrm{KBr}, v, \mathrm{~cm}^{-1}\right): 3004,1644,1610,1511,1477,1456,1415$, 1385, 1301, 1254;

HRMS (ESI): $\mathrm{m} / \mathrm{z}$ calcd for: $\mathrm{C}_{27} \mathrm{H}_{22} \mathrm{NO}_{2}, 392.1651[\mathrm{M}+\mathrm{H}]^{+}$; found: 392.1641 .

\section{General Procedure for the Synthesis of 8a-8s}

\section{7-Phenyl-7H-acenaphtho[1,2-b]indol-11-yl acetate (8a)}

In a typical experiment procedure, 3-(phenylamino)cyclohex-2enone $7 \mathbf{a}$ (1.0 mmol, $0.187 \mathrm{~g}, 1.0$ equiv) and acenaphthylene-1,2dione 2 ( $1.0 \mathrm{mmol}, 0.182 \mathrm{~g}, 1.0$ equiv) were introduced in a 10$\mathrm{mL}$ Initiator ${ }^{\mathrm{TM}}$ reaction vial, acetic anhydride $(1.5 \mathrm{~mL})$ was then successively added. Subsequently, the reaction vial was capped and then pre-stirring for 20 second. The mixture was irradiated (Time: $20 \mathrm{~min}$, Temperature: $120{ }^{\circ} \mathrm{C}$; Absorption Level: High; Fixed Hold Time) until TLC revealed that conversion of the starting materials were completed. The reaction mixture was then cooled to room temperature and then diluted with cold water (25 
$\mathrm{mL}$ ). The solid product was collected by Büchner filtration to give pure esterification indoles $\mathbf{8 a}$.

An orange solid: $0.274 \mathrm{~g}$, yield $73 \%$; Mp: $197-199^{\circ} \mathrm{C}$;

${ }^{1} \mathrm{H}$ NMR (400 MHz, DMSO- $\left.d_{6}\right)(\delta, \mathrm{ppm}): 7.89$ (d, $1 \mathrm{H}, J=8.0$ $\mathrm{Hz}, \mathrm{ArH}), 7.84(\mathrm{~d}, 2 \mathrm{H}, J=7.6 \mathrm{~Hz}, \mathrm{ArH}), 7.79(\mathrm{~d}, 1 \mathrm{H}, J=3.2 \mathrm{~Hz}$, ArH), 7.77-7.75 (m, 2H, ArH), $7.74(\mathrm{~d}, 1 \mathrm{H}, J=3.6 \mathrm{~Hz}, \mathrm{ArH})$, $7.65(\mathrm{t}, 2 \mathrm{H}, J=8.0 \mathrm{~Hz}, \mathrm{ArH}), 7.52(\mathrm{t}, 1 \mathrm{H}, J=7.2 \mathrm{~Hz}, \mathrm{ArH}), 7.38$ $(\mathrm{d}, 1 \mathrm{H}, J=6.8 \mathrm{~Hz}, \mathrm{ArH}), 7.29(\mathrm{~d}, 1 \mathrm{H}, J=8.4 \mathrm{~Hz}, \mathrm{ArH}), 7.22(\mathrm{t}$, $1 \mathrm{H}, J=8.0 \mathrm{~Hz}, \mathrm{ArH}), 7.07$ (d, 1H, $J=7.6 \mathrm{~Hz}, \mathrm{ArH}), 2.63$ (s, 3H, $\mathrm{CH}_{3}$;

${ }^{13} \mathrm{C}$ NMR $\left(100 \mathrm{MHz}, \mathrm{DMSO}-d_{6}\right)(\delta, \mathrm{ppm}): 169.8,144.4,143.6$, $143.4,137.4,132.4,132.2,130.8,129.6,129.0,128.6,128.3$, $128.0,126.3,125.8,123.3,121.6,121.5,118.2,117.3,115.1$, 109.6, 22.0;

IR $\left(\mathrm{KBr}, \mathrm{v}, \mathrm{cm}^{-1}\right): 3045,1763,1743,1638,1609,1596,1568$, 1501, 1450, 1369, 1339;

HRMS (ESI): $\mathrm{m} / \mathrm{z}$ calcd for: $\mathrm{C}_{26} \mathrm{H}_{17} \mathrm{NNaO}_{2}, 398.1157$ [M+Na $]^{+}$; found: 398.1166 .

\section{7-(4-Chlorophenyl)-7H-acenaphtho[1,2-b]indol-11-yl acetate (8b)}

An orange solid: $0.311 \mathrm{~g}$, yield $76 \%$; Mp: $183-185^{\circ} \mathrm{C}$;

${ }^{1} \mathrm{H}$ NMR (400 MHz, DMSO- $\left.d_{6}\right)(\delta, \mathrm{ppm}): 7.91-7.89$ (m, 2H, ArH), 7.88 (s, 1H, ArH), 7.82 (s, 1H, ArH), 7.80 (s, 1H, ArH), $7.78(\mathrm{~s}, 1 \mathrm{H}, \mathrm{ArH}), 7.74(\mathrm{~d}, 1 \mathrm{H}, J=6.8 \mathrm{~Hz}, \mathrm{ArH}), 7.65(\mathrm{t}, 1 \mathrm{H}, J=$ $7.2 \mathrm{~Hz}, \mathrm{ArH}), 7.54$ (t, 1H, $J=7.2 \mathrm{~Hz}, \operatorname{ArH}), 7.43(\mathrm{~d}, 1 \mathrm{H}, J=7.2$ $\mathrm{Hz}, \operatorname{ArH}), 7.31(\mathrm{~d}, 1 \mathrm{H}, J=8.0 \mathrm{~Hz}, \mathrm{ArH}), 7.24(\mathrm{t}, 1 \mathrm{H}, J=7.6 \mathrm{~Hz}$, ArH), 7.08 (d, $1 \mathrm{H}, J=7.6 \mathrm{~Hz}, \mathrm{ArH}), 2.63\left(\mathrm{~s}, 3 \mathrm{H}, \mathrm{CH}_{3}\right)$;

${ }^{13} \mathrm{C}$ NMR (100 MHz, DMSO- $\left.d_{6}\right)(\delta, \mathrm{ppm}): 169.8,144.2,143.5$, $143.4,136.3,133.3,132.3,132.1,130.8,129.5,129.0,128.7$, $128.2,128.1(2), 128.0(6), 125.9,123.5,121.8,121.6,118.5$, 117.3, 115.3, 109.6, 22.0;

IR $\left(\mathrm{KBr}, \mathrm{v}, \mathrm{cm}^{-1}\right)$ : 3055, 1760, 1654, 1613, 1522, 1498, 1479, 1392, 1356, 1329;

HRMS (ESI): $\mathrm{m} / \mathrm{z}$ calcd for: $\mathrm{C}_{26} \mathrm{H}_{16} \mathrm{ClNNaO}_{2}, \quad 432.0767$ $[\mathrm{M}+\mathrm{Na}]^{+}$; found: 432.0754 .

\section{7-(4-Chlorophenyl)-9-methyl-7H-acenaphtho[1,2-b]indol-11-} yl acetate $(8 c)$

An orange solid: $0.326 \mathrm{~g}$, yield $77 \%$; Mp: $177-179^{\circ} \mathrm{C}$;

${ }^{1} \mathrm{H}$ NMR (400 MHz, DMSO- $\left.d_{6}\right)(\delta, \mathrm{ppm}): 7.89-7.85(\mathrm{~m}, 3 \mathrm{H}$, ArH), 7.82-7.81 (m, 1H, ArH), 7.80-7.79 (m, 1H, ArH), 7.77 (d, $1 \mathrm{H}, J=8.4 \mathrm{~Hz}, \mathrm{ArH}), 7.70(\mathrm{~d}, 1 \mathrm{H}, J=6.4 \mathrm{~Hz}, \mathrm{ArH}), 7.65-7.61$ $(\mathrm{m}, 1 \mathrm{H}, \operatorname{ArH}), 7.52(\mathrm{t}, 1 \mathrm{H}, J=6.8 \mathrm{~Hz}, \mathrm{ArH}), 7.38(\mathrm{~d}, 1 \mathrm{H}, J=6.8$ $\mathrm{Hz}, \mathrm{ArH}), 7.12(\mathrm{~s}, 1 \mathrm{H}, \mathrm{ArH}), 6.92(\mathrm{~s}, 1 \mathrm{H}, \mathrm{ArH}), 2.61(\mathrm{~s}, 3 \mathrm{H}$, $\left.\mathrm{CH}_{3}\right), 2.42\left(\mathrm{~s}, 3 \mathrm{H}, \mathrm{CH}_{3}\right)$;

${ }^{13} \mathrm{C}$ NMR $\left(100 \mathrm{MHz}, \mathrm{DMSO}-d_{6}\right)(\delta, \mathrm{ppm}): 169.8,143.7,143.1$, $136.4,133.5,133.2,132.3,131.9,130.8,129.5,129.0,128.4$, $128.3,128.2,128.0,125.8,121.5(3), 121.5(0), 118.5,116.7$, 115.3, 109.4, 21.9, 21.7;

IR $\left(\mathrm{KBr}, v, \mathrm{~cm}^{-1}\right): 3059,1764,1629,1609,1581,1496,1439$, 1417, 1366, 1346, 1335;

HRMS (ESI): $\mathrm{m} / \mathrm{z}$ calcd for: $\mathrm{C}_{27} \mathrm{H}_{18} \mathrm{ClNNaO}_{2}, \quad 446.0924$ $[\mathrm{M}+\mathrm{Na}]^{+}$; found: 446.0937 .

\section{7-(4-Methoxyphenyl)-9-methyl-7H-acenaphtho[1,2-b]indol-} 11-yl acetate (8d)

An orange solid: $0.310 \mathrm{~g}$, yield $74 \%$; Mp: $185-187^{\circ} \mathrm{C}$;

${ }^{1} \mathrm{H}$ NMR (400 MHz, DMSO- $\left.d_{6}\right)(\delta, \mathrm{ppm}): 7.85$ (d, $1 \mathrm{H}, J=8.0$ $\mathrm{Hz}, \mathrm{ArH}), 7.75-7.66(\mathrm{~m}, 4 \mathrm{H}, \mathrm{ArH}), 7.61$ (t, $1 \mathrm{H}, J=8.0 \mathrm{~Hz}, \mathrm{ArH})$, 7.49 (t, $1 \mathrm{H}, J=7.2 \mathrm{~Hz}, \operatorname{ArH}), 7.29-7.27(\mathrm{~m}, 3 \mathrm{H}, \operatorname{ArH}), 7.02$ (s, $1 \mathrm{H}, \mathrm{ArH}), 6.88(\mathrm{~s}, 1 \mathrm{H}, \mathrm{ArH}), 3.92\left(\mathrm{~s}, 3 \mathrm{H}, \mathrm{OCH}_{3}\right), 2.61(\mathrm{~s}, 3 \mathrm{H}$, $\left.\mathrm{CH}_{3}\right), 2.40\left(\mathrm{~s}, 3 \mathrm{H}, \mathrm{CH}_{3}\right)$;

${ }^{13} \mathrm{C}$ NMR $\left(100 \mathrm{MHz}, \mathrm{DMSO}-d_{6}\right)(\delta, \mathrm{ppm}): 169.8,159.5,144.3$, 144.2 , 143.0, 133.0, 132.6, 132.0, 130.1, 129.5, 129.0, 128.6,
$128.3,128.0,127.8,125.5,121.2,117.6,116.3,115.8,115.1$, 109.4, 100.0, 56.0, 21.9, 21.7;

IR $\left(\mathrm{KBr}, v, \mathrm{~cm}^{-1}\right): 3057,1766,1609,1569,1514,1479,1443$, 1366, 1335, 1301;

HRMS (ESI): m/z calcd for: $\mathrm{C}_{28} \mathrm{H}_{21} \mathrm{NNaO}_{3}, 442.1419$ [M+Na] $]^{+}$; found: 442.1402 .

7-(4-Chlorophenyl)-9-phenyl-7H-acenaphtho[1,2-b]indol-11yl acetate (8e)

An orange solid: $0.388 \mathrm{~g}$, yield $80 \%$; Mp: $223-225^{\circ} \mathrm{C}$;

${ }^{1} \mathrm{H}$ NMR (400 MHz, DMSO- $\left.d_{6}\right)(\delta, \mathrm{ppm}):$ 7.95-7.89 $(\mathrm{m}, 3 \mathrm{H}$, ArH), $7.80(\mathrm{t}, 3 \mathrm{H}, J=7.6 \mathrm{~Hz}, \operatorname{ArH}), 7.75(\mathrm{~d}, 1 \mathrm{H}, J=6.4 \mathrm{~Hz}$, ArH), 7.70-7.63 (m, 3H, ArH), 7.55-7.41 (m, 6H, ArH), $7.36(\mathrm{t}$, $1 \mathrm{H}, J=7.2 \mathrm{~Hz}, \mathrm{ArH}), 2.65\left(\mathrm{~s}, 3 \mathrm{H}, \mathrm{CH}_{3}\right)$;

${ }^{13} \mathrm{C}$ NMR (100 MHz, DMSO- $\left.d_{6}\right)(\delta, \mathrm{ppm}): 169.9,144.9,144.0$, $143.7,140.4,136.2,136.1,133.3,132.2,132.1,130.9,129.6$, $129.4,129.0,128.8,128.3,128.1,127.8,127.3,126.0,121.9$, 121.7, 118.4, 116.6, 114.5, 107.4, 22.0;

IR $\left(\mathrm{KBr}, v, \mathrm{~cm}^{-1}\right): 3036,1647,1609,1522,1489,1473,1457$, 1402, 1360, 1347, 1328, 1297;

HRMS (ESI): $\mathrm{m} / \mathrm{z}$ calcd for: $\mathrm{C}_{32} \mathrm{H}_{20} \mathrm{ClNNaO}_{2}, \quad 508.1080$ $[\mathrm{M}+\mathrm{Na}]^{+}$; found: 508.1093 .

\section{7-(4-Bromophenyl)-9-phenyl-7H-acenaphtho[1,2-b]indol-11-} yl acetate (8f)

An orange solid: $0.407 \mathrm{~g}$, yield $77 \%$; Mp: $242-244{ }^{\circ} \mathrm{C}$;

${ }^{1} \mathrm{H}$ NMR (400 MHz, DMSO- $\left.d_{6}\right)(\delta, \mathrm{ppm}):$ 7.95-7.86 (m, 5H, $\operatorname{ArH}), 7.79(\mathrm{~d}, 1 \mathrm{H}, J=8.0 \mathrm{~Hz}, \operatorname{ArH}), 7.75(\mathrm{~d}, 1 \mathrm{H}, J=6.8 \mathrm{~Hz}$, ArH), 7.70-7.64 (m, 3H, ArH), 7.55-7.51 (m, 2H, ArH), 7.48$7.43(\mathrm{~m}, 4 \mathrm{H}, \mathrm{ArH}), 7.36$ (t, 1H, $J=7.2 \mathrm{~Hz}, \operatorname{ArH}), 2.65$ (s, 3H, $\mathrm{CH}_{3}$;

${ }^{13} \mathrm{C}$ NMR (100 MHz, DMSO- $\left.d_{6}\right)(\delta, \mathrm{ppm}): 169.9,144.8,143.9$, $143.7,140.4,136.6,136.1,133.8,132.2,132.1,129.6,129.4$, $129.0,128.8,128.5,128.1,127.8,127.3,126.0,121.9,121.8$, $118.5,116.6,114.5,107.4,22.0$;

IR $\left(\mathrm{KBr}, v, \mathrm{~cm}^{-1}\right): 3054,1760,1609,1598,1564,1496,1447$, 1434, 1398, 1365, 1346, 1298;

HRMS (ESI): $\mathrm{m} / \mathrm{z}$ calcd for: $\mathrm{C}_{32} \mathrm{H}_{20} \mathrm{BrNNaO}_{2}, \quad 552.0575$ $[\mathrm{M}+\mathrm{Na}]^{+}$; found: 552.0598 .

\section{9-Phenyl-7-( $p$-tolyl)-7H-acenaphtho[1,2-b]indol-11-yl acetate} (8g)

An orange solid: $0.344 \mathrm{~g}$, yield $74 \%$; Mp: $218-220^{\circ} \mathrm{C}$;

${ }^{1} \mathrm{H}$ NMR (400 MHz, DMSO- $\left.d_{6}\right)(\delta, \mathrm{ppm}): 7.88$ (d, $1 \mathrm{H}, J=8.0$ $\mathrm{Hz}, \mathrm{ArH}), 7.75$ (t, 4H, $J=9.2 \mathrm{~Hz}, \operatorname{ArH}), 7.65(\mathrm{t}, 3 \mathrm{H}, J=7.6 \mathrm{~Hz}$, ArH), 7.55-7.49 (m, 3H, ArH), 7.47-7.40 (m, 4H, ArH), 7.37$7.33(\mathrm{~m}, 2 \mathrm{H}, \mathrm{ArH}), 2.65\left(\mathrm{~s}, 3 \mathrm{H}, \mathrm{CH}_{3}\right), 2.49\left(\mathrm{~s}, 3 \mathrm{H}, \mathrm{CH}_{3}\right)$;

${ }^{13} \mathrm{C}$ NMR (100 MHz, DMSO- $\left.d_{6}\right)(\delta, \mathrm{ppm}): 169.9,145.2,144.1$, $143.7,140.5,138.6,135.8,134.7,132.3,131.3,129.6,129.4$, $129.0,128.6,128.3,128.0,127.7,127.2,126.2,125.8,121.7$, $121.5,117.9,116.5,114.2,107.5,22.0,21.3$;

IR $\left(\mathrm{KBr}, \mathrm{v}, \mathrm{cm}^{-1}\right)$ : 3050, 1762, 1610, 1597, 1565, 1517, 1479, 1448, 1436, 1420, 1364, 1334;

HRMS (ESI): $\mathrm{m} / \mathrm{z}$ calcd for: $\mathrm{C}_{33} \mathrm{H}_{23} \mathrm{NNaO}_{2}, 488.1626[\mathrm{M}+\mathrm{Na}]^{+}$; found: 488.1641 .

7-Benzyl-9-phenyl-7H-acenaphtho[1,2-b]indol-11-yl acetate (8h)

An orange solid: $0.307 \mathrm{~g}$, yield $66 \%$; Mp: $223-225^{\circ} \mathrm{C}$;

${ }^{1} \mathrm{H}$ NMR (400 MHz, DMSO- $\left.d_{6}\right)(\delta, \mathrm{ppm}): 7.94(\mathrm{~d}, 2 \mathrm{H}, J=6.8$ $\mathrm{Hz}, \mathrm{ArH}), 7.88(\mathrm{~d}, 1 \mathrm{H}, J=8.4 \mathrm{~Hz}, \mathrm{ArH}), 7.76(\mathrm{t}, 3 \mathrm{H}, J=8.0 \mathrm{~Hz}$, $\mathrm{ArH}), 7.69(\mathrm{~d}, 1 \mathrm{H}, J=6.0 \mathrm{~Hz}, \mathrm{ArH}), 7.65-7.56(\mathrm{~m}, 2 \mathrm{H}, \mathrm{ArH})$, $7.49(\mathrm{t}, 2 \mathrm{H}, J=7.2 \mathrm{~Hz}, \mathrm{ArH}), 7.37-7.30(\mathrm{~m}, 6 \mathrm{H}, \mathrm{ArH}), 7.25-7.24$ (m, 1H, ArH), 5.96 (s, 2H, $\left.\mathrm{CH}_{2}\right), 2.63\left(\mathrm{~s}, 3 \mathrm{H}, \mathrm{CH}_{3}\right)$;

${ }^{13} \mathrm{C}$ NMR (100 MHz, DMSO- $\left.d_{6}\right)(\delta, \mathrm{ppm}): 169.9,145.8,144.3$, $143.6,140.6,137.9,135.0,132.7,132.3,129.5,129.4,129.3$, 
$129.0,128.4,128.1,128.0,127.6,127.2,127.1,125.4,122.2$, 121.0, 117.0, 116.2, 113.4, 107.7, 48.6, 22.0;

IR $\left(\mathrm{KBr}, v, \mathrm{~cm}^{-1}\right): 3030,1763,1609,1564,1498,1452,1436$, 1417, 1369, 1355, 1335;

HRMS (ESI): $\mathrm{m} / \mathrm{z}$ calcd for: $\mathrm{C}_{33} \mathrm{H}_{23} \mathrm{NNaO}_{2}, 488.1626[\mathrm{M}+\mathrm{Na}]^{+}$; found: 488.1611 .

\section{7-(4-Chlorophenyl)-7H-acenaphtho[1,2-b]indol-11-yl propionate $(8 \mathrm{i})$}

A red-orange solid: $0.300 \mathrm{~g}$, yield $71 \%$; $\mathrm{Mp}: 155-157^{\circ} \mathrm{C}$;

${ }^{1} \mathrm{H}$ NMR $\left(400 \mathrm{MHz}, \mathrm{DMSO}-d_{6}\right)(\delta, \mathrm{ppm}): 7.91-7.87(\mathrm{~m}, 3 \mathrm{H}$, ArH), 7.81-7.77 (m, 3H, ArH), $7.70(\mathrm{~d}, 1 \mathrm{H}, J=6.8 \mathrm{~Hz}, \mathrm{ArH})$, $7.64(\mathrm{t}, 1 \mathrm{H}, J=7.6 \mathrm{~Hz}, \mathrm{ArH}), 7.53(\mathrm{t}, 1 \mathrm{H}, J=7.2 \mathrm{~Hz}, \operatorname{ArH}), 7.42$ $(\mathrm{d}, 1 \mathrm{H}, J=6.8 \mathrm{~Hz}, \operatorname{ArH}), 7.30(\mathrm{~d}, 1 \mathrm{H}, J=8.0 \mathrm{~Hz}, \operatorname{ArH}), 7.23(\mathrm{t}$, $1 \mathrm{H}, J=8.0 \mathrm{~Hz}, \mathrm{ArH}), 7.08(\mathrm{~d}, 1 \mathrm{H}, J=7.6 \mathrm{~Hz}, \mathrm{ArH}), 3.02-2.97$ $\left(\mathrm{m}, 2 \mathrm{H}, \mathrm{CH}_{2}\right), 1.28\left(\mathrm{t}, 3 \mathrm{H}, J=7.6 \mathrm{~Hz}, \mathrm{CH}_{3}\right)$

${ }^{13} \mathrm{C}$ NMR (100 MHz, DMSO- $\left.d_{6}\right)(\delta, \mathrm{ppm}): 173.2,144.2$, $143.5(3), 143.5(0), 136.3,133.3,132.3,132.1,130.8,129.6$, $129.0,128.7,128.2,128.1(3), 128.0(6), 125.9,123.5,121.8$, $121.6,118.5,117.3,115.2,109.5,27.9,9.5$;

IR $\left(\mathrm{KBr}, v, \mathrm{~cm}^{-1}\right): 3053,1752,1608,1595,1568,1498,1450$, 1420, 1401, 1339;

HRMS (ESI): $\mathrm{m} / \mathrm{z}$ calcd for: $\mathrm{C}_{27} \mathrm{H}_{18} \mathrm{ClNNaO}_{2}, 446.0924$ $[\mathrm{M}+\mathrm{Na}]^{+}$; found: 446.0936 .

\section{7-(4-Bromophenyl)-9-methyl-7H-acenaphtho[1,2-b]indol-11-} yl propionate $(8 \mathbf{j})$

A red solid: $0.332 \mathrm{~g}$, yield $69 \%$; Mp: $195-197{ }^{\circ} \mathrm{C}$;

${ }^{1} \mathrm{H}$ NMR (400 MHz, DMSO- $\left.d_{6}\right)(\delta, \mathrm{ppm}): 7.93$ (d, 2H, $J=8.4$ $\mathrm{Hz}, \mathrm{ArH}), 7.87(\mathrm{~d}, 1 \mathrm{H}, J=8.0 \mathrm{~Hz}, \mathrm{ArH}), 7.80-7.75(\mathrm{~m}, 3 \mathrm{H}$, ArH), 7.67-7.60 (m, 2H, ArH), $7.51(\mathrm{t}, 1 \mathrm{H}, J=7.2 \mathrm{~Hz}, \operatorname{ArH})$, $7.39(\mathrm{~d}, 1 \mathrm{H}, J=6.8 \mathrm{~Hz}, \operatorname{ArH}), 7.12(\mathrm{~s}, 1 \mathrm{H}, \operatorname{ArH}), 6.92(\mathrm{~s}, 1 \mathrm{H}$, ArH), 3.01-2.95 (m, 2H, $\left.\mathrm{CH}_{2}\right), 2.41\left(\mathrm{~s}, 3 \mathrm{H}, \mathrm{CH}_{3}\right), 1.28(\mathrm{t}, 3 \mathrm{H}, J=$ $\left.7.2 \mathrm{~Hz}, \mathrm{CH}_{3}\right)$;

${ }^{13} \mathrm{C}$ NMR (100 MHz, DMSO- $\left.d_{6}\right)(\delta, \mathrm{ppm}): 173.2,143.6(4)$, $143.6(0), 143.1,136.8,133.7,133.6,132.3,131.9,129.5,128.9$, $128.5,128.4,128.3,128.0,125.9,121.6,121.5,118.6,116.6$, $115.3,109.4,27.9,21.7,9.5$

IR $\left(\mathrm{KBr}, v, \mathrm{~cm}^{-1}\right): 3034,1754,1622,1608,1583,1494,1467$, 1442, 1413, 1396, 1375, 1345;

HRMS (ESI): $\mathrm{m} / \mathrm{z}$ calcd for: $\mathrm{C}_{28} \mathrm{H}_{20} \mathrm{BrNNaO}_{2}, \quad 504.0575$ $[\mathrm{M}+\mathrm{Na}]^{+}$; found: 504.0589 .

\section{9-Methyl-7-(p-tolyl)-7H-acenaphtho[1,2-b]indol-11-yl propionate (8k) \\ A red solid: $0.354 \mathrm{~g}$, yield $85 \%$; Mp: $140-142{ }^{\circ} \mathrm{C}$;}

${ }^{1} \mathrm{H}$ NMR $\left(400 \mathrm{MHz}, \mathrm{DMSO}-d_{6}\right)(\delta, \mathrm{ppm}): 7.84(\mathrm{~d}, 1 \mathrm{H}, J=8.0$ $\mathrm{Hz}, \mathrm{ArH}), 7.73(\mathrm{~d}, 1 \mathrm{H}, J=8.0 \mathrm{~Hz}, \mathrm{ArH}), 7.68-7.59(\mathrm{~m}, 4 \mathrm{H}$, $\operatorname{ArH}), 7.54(\mathrm{~d}, 2 \mathrm{H}, J=8.0 \mathrm{~Hz}, \operatorname{ArH}), 7.49(\mathrm{t}, 1 \mathrm{H}, J=7.2 \mathrm{~Hz}$, ArH), 7.32 (d, 1H, $J=6.8 \mathrm{~Hz}, \mathrm{ArH}), 7.06$ (s, 1H, ArH), 6.89 (s, $1 \mathrm{H}, \mathrm{ArH}), 3.00-2.95\left(\mathrm{~m}, 2 \mathrm{H}, \mathrm{CH}_{2}\right), 2.49\left(\mathrm{~s}, 3 \mathrm{H}, \mathrm{CH}_{3}\right), 2.40(\mathrm{~s}$, $\left.3 \mathrm{H}, \mathrm{CH}_{3}\right), 1.28\left(\mathrm{t}, 3 \mathrm{H}, J=7.2 \mathrm{~Hz}, \mathrm{CH}_{3}\right)$;

${ }^{13} \mathrm{C}$ NMR $\left(100 \mathrm{MHz}, \mathrm{DMSO}-d_{6}\right)(\delta, \mathrm{ppm}): 173.2,144.0,143.9$, $143.1,138.5,134.9,133.2,132.6,132.0,131.2,129.5,128.9$, $128.6,128.3,128.0,126.1,125.6,121.3,121.2,118.0,116.3$, $115.2,109.4,27.9,21.7,21.3,9.5$;

IR $\left(\mathrm{KBr}, v, \mathrm{~cm}^{-1}\right): 3042,1741,1660,1609,1515,1462,1446$, 1410, 1391, 1349;

HRMS (ESI): $\mathrm{m} / \mathrm{z}$ calcd for: $\mathrm{C}_{29} \mathrm{H}_{23} \mathrm{NNaO}_{2}, 440.1626[\mathrm{M}+\mathrm{Na}]^{+}$; found: 440.1612 .

\section{7-(4-Chlorophenyl)-9-phenyl-7H-acenaphtho[1,2-b]indol-11-} yl propionate $(81)$

A red-orange solid: $0.369 \mathrm{~g}$, yield $74 \%$; Mp: $219-221^{\circ} \mathrm{C}$;

${ }^{1} \mathrm{H}$ NMR $\left(400 \mathrm{MHz}, \mathrm{DMSO}-d_{6}\right)(\delta, \mathrm{ppm}): 7.97-7.91(\mathrm{~m}, 3 \mathrm{H}$, ArH), 7.83-7.79 (m, 3H, ArH), 7.71-7.64 (m, 4H, ArH), 7.56- $7.44(\mathrm{~m}, 6 \mathrm{H}, \mathrm{ArH}), 7.38-7.35(\mathrm{~m}, 1 \mathrm{H}, \mathrm{ArH}), 3.05-3.02(\mathrm{~m}, 2 \mathrm{H}$, $\left.\mathrm{CH}_{2}\right), 1.31\left(\mathrm{t}, 3 \mathrm{H}, J=6.4 \mathrm{~Hz}, \mathrm{CH}_{3}\right)$;

${ }^{13} \mathrm{C}$ NMR $\left(100 \mathrm{MHz}\right.$, DMSO- $\left.d_{6}\right)(\delta, \mathrm{ppm}): 173.3,144.9,144.0$, $143.8,140.4,136.2,136.1,133.3,132.2,132.1,130.9,129.6$, $129.4,129.0,128.8,128.3,128.1,127.8,127.3,126.0,121.9$, $121.7,118.4,116.6,114.5,107.4,28.0,9.5$;

IR $\left(\mathrm{KBr}, \mathrm{v}, \mathrm{cm}^{-1}\right): 3045,1749,1608,1597,1564,1501,1449$, 1437, 1418, 1377, 1344;

HRMS (ESI): $\mathrm{m} / \mathrm{z}$ calcd for: $\mathrm{C}_{33} \mathrm{H}_{22} \mathrm{ClNNaO}_{2}, \quad 522.1237$ $[\mathrm{M}+\mathrm{Na}]^{+}$; found: 522.1249 .

\section{7-(3,5-Dichlorophenyl)-9-phenyl-7H-acenaphtho[1,2-b]indol-} 11-yl propionate $(8 \mathrm{~m})$

A red solid: $0.400 \mathrm{~g}$, yield $75 \%$; Mp: $194-196{ }^{\circ} \mathrm{C}$;

${ }^{1} \mathrm{H}$ NMR (400 MHz, DMSO- $\left.d_{6}\right)(\delta, \mathrm{ppm}): 8.09$ (s, 2H, ArH), 7.93-7.91 (m, 2H, ArH), 7.81 (d, $1 \mathrm{H}, J=8.4 \mathrm{~Hz}, \mathrm{ArH}), 7.74-7.64$ (m, 4H, ArH), 7.59-7.53 (m, 2H, ArH), 7.49-7.44 (m, 3H, ArH), 7.41-7.35 (m, 2H, ArH), 3.05-3.00 (m, 2H, $\left.\mathrm{CH}_{2}\right), 1.30(\mathrm{t}, 3 \mathrm{H}, J=$ 7.6 Hz, $\left.\mathrm{CH}_{3}\right)$;

${ }^{13} \mathrm{C}$ NMR (100 MHz, DMSO- $\left.d_{6}\right)(\delta, \mathrm{ppm}): 173.3,144.7,144.0$, $143.7,140.4,139.6,136.3,135.9,132.1,132.0,129.6,129.4$, $129.0,128.8,128.2,128.1,127.8,127.4,126.2,125.8,121.9$, $121.7,118.9,116.6,114.8,107.5,28.0,9.5$;

IR $\left(\mathrm{KBr}, v, \mathrm{~cm}^{-1}\right): 3045,1741,1666,1610,1586,1571,1523$, 1509, 1472, 1458, 1431, 1394, 1368, 1352;

HRMS (ESI): $\mathrm{m} / \mathrm{z}$ calcd for: $\mathrm{C}_{33} \mathrm{H}_{21} \mathrm{Cl}_{2} \mathrm{NNaO}_{2}, \quad 556.0847$ $[\mathrm{M}+\mathrm{Na}]^{+}$; found: 556.0862 .

\section{7-(4-Bromophenyl)-9-phenyl-7H-acenaphtho[1,2-b]indol-11-} yl propionate $(8 n)$

A red-orange solid: $0.380 \mathrm{~g}$, yield $70 \%$; Mp: $197-199{ }^{\circ} \mathrm{C}$;

${ }^{1} \mathrm{H}$ NMR $\left(400 \mathrm{MHz}, \mathrm{DMSO}-d_{6}\right)(\delta, \mathrm{ppm}): 7.97-7.89(\mathrm{~m}, 5 \mathrm{H}$, ArH), $7.81(\mathrm{~d}, 1 \mathrm{H}, J=8.0 \mathrm{~Hz}, \mathrm{ArH}), 7.74-7.64(\mathrm{~m}, 4 \mathrm{H}, \mathrm{ArH})$, 7.57-7.52 (m, 2H, ArH), 7.49-7.44 (m, 4H, ArH), $7.36(\mathrm{t}, 1 \mathrm{H}, J=$ $6.8 \mathrm{~Hz}, \mathrm{ArH}), 3.05-3.00\left(\mathrm{~m}, 2 \mathrm{H}, \mathrm{CH}_{2}\right), 1.31(\mathrm{t}, 3 \mathrm{H}, J=7.2 \mathrm{~Hz}$, $\mathrm{CH}_{3}$ );

${ }^{13} \mathrm{C}$ NMR (100 MHz, DMSO- $\left.d_{6}\right)(\delta, \mathrm{ppm}): 173.3,144.8,143.9$, $143.8,140.4,136.6,136.1,133.8,132.2,132.1,129.6,129.4$, $129.0,128.8,128.6,128.1(3), 128.0(9), 127.8,127.3,126.0$, $121.9,121.8,121.7,118.5,116.6,114.5,107.4,28.0,9.5$;

IR $\left(\mathrm{KBr}, v, \mathrm{~cm}^{-1}\right): 3045,1749,1608,1597,1564,1500,1449$, 1437, 1419, 1399, 1345;

HRMS (ESI): $\mathrm{m} / \mathrm{z}$ calcd for: $\mathrm{C}_{33} \mathrm{H}_{22} \mathrm{BrNNaO}_{2}, \quad 566.0732$ $[\mathrm{M}+\mathrm{Na}]^{+}$; found: 566.0755 .

\section{9-Phenyl-7-(p-tolyl)-7H-acenaphtho[1,2-b]indol-11-yl propionate $(80)$}

A red-orange solid: $0.374 \mathrm{~g}$, yield $78 \%$; Mp: $167-169^{\circ} \mathrm{C}$;

${ }^{1} \mathrm{H}$ NMR $\left(400 \mathrm{MHz}, \mathrm{DMSO}-d_{6}\right)(\delta, \mathrm{ppm}): 7.89(\mathrm{~d}, 1 \mathrm{H}, J=8.0$ $\mathrm{Hz}, \mathrm{ArH}), 7.80-7.75(\mathrm{~m}, 3 \mathrm{H}, \mathrm{ArH}), 7.71-7.62(\mathrm{~m}, 4 \mathrm{H}, \mathrm{ArH})$, 7.57-7.50 (m, 3H, ArH), 7.47-7.44 (m, 3H, ArH), 7.40-7.33 (m, $3 \mathrm{H}, \mathrm{ArH}), 3.05-2.99\left(\mathrm{~m}, 2 \mathrm{H}, \mathrm{CH}_{2}\right), 2.50\left(\mathrm{~s}, 3 \mathrm{H}, \mathrm{CH}_{3}\right), 1.31(\mathrm{t}, 3 \mathrm{H}$, $\left.J=7.6 \mathrm{~Hz}, \mathrm{CH}_{3}\right)$;

${ }^{13} \mathrm{C}$ NMR (100 MHz, DMSO- $\left.d_{6}\right)(\delta, \mathrm{ppm}): 173.3,145.2,144.1$, $143.8,140.5,138.7,135.8,134.7,132.3(3), 132.2(9), 131.3$, $129.6,129.4,129.0,128.6,128.3,128.0,127.7,127.2,126.2$, $125.8,121.7,121.4,117.9,116.5,114.2,107.4,28.0,21.3,9.5$; IR $\left(\mathrm{KBr}, \mathrm{v}, \mathrm{cm}^{-1}\right): 3032,1750,1607,1565,1517,1483,1448$, 1436, 1418, 1379, 1340;

HRMS (ESI): $\mathrm{m} / \mathrm{z}$ calcd for: $\mathrm{C}_{34} \mathrm{H}_{25} \mathrm{NNaO}_{2}, 502.1783[\mathrm{M}+\mathrm{Na}]^{+}$; found: 502.1794 .

\section{7-Benzyl-9-phenyl-7H-acenaphtho[1,2-b]indol-11-yl} propionate $(8 p)$

A red solid: $0.321 \mathrm{~g}$, yield $67 \%$; Mp: $219-221^{\circ} \mathrm{C}$;

${ }^{1} \mathrm{H}$ NMR (400 MHz, DMSO- $\left.d_{6}\right)(\delta, \mathrm{ppm}): 7.97-7.89(\mathrm{~m}, 3 \mathrm{H}$, ArH), 7.78-7.74 (m, 3H, ArH), 7.65-7.58 (m, 3H, ArH), 7.49 (t, 
$2 \mathrm{H}, J=7.6 \mathrm{~Hz}, \mathrm{ArH}), 7.38-7.26(\mathrm{~m}, 6 \mathrm{H}, \mathrm{ArH}), 7.26-7.22(\mathrm{~m}$, $1 \mathrm{H}, \mathrm{ArH}), 5.98\left(\mathrm{~s}, 2 \mathrm{H}, \mathrm{CH}_{2}\right), 3.02-2.97\left(\mathrm{~m}, 2 \mathrm{H}, \mathrm{CH}_{2}\right), 1.29(\mathrm{t}, 3 \mathrm{H}$, $\left.J=7.6 \mathrm{~Hz}, \mathrm{CH}_{3}\right)$;

${ }^{13} \mathrm{C}$ NMR (100 MHz, DMSO- $\left.d_{6}\right)(\delta, \mathrm{ppm}): 173.3,145.7,144.3$, $143.6,140.5,137.9,135.0,132.7,132.2,129.5,129.4,129.2$, $128.9,128.4,128.1,128.0,127.6,127.2,127.1,125.4,122.2$, 120.9, 117.0, 116.1, 113.3, 107.7, 48.6, 27.9, 9.5;

IR $\left(\mathrm{KBr}, v, \mathrm{~cm}^{-1}\right): 3028,1759,1731,1649,1609,1568,1497$, 1477, 1452, 1431, 1418, 1354, 1333;

HRMS (ESI): $\mathrm{m} / \mathrm{z}$ calcd for: $\mathrm{C}_{34} \mathrm{H}_{25} \mathrm{NNaO}_{2}, 502.1783[\mathrm{M}+\mathrm{Na}]^{+}$; found: 502.1796 .

\section{7-(4-Chlorophenyl)-9-phenyl-7H-acenaphtho[1,2-b]indol-11- yl butyrate (8q)}

A red-orange solid: $0.354 \mathrm{~g}$, yield $69 \%$; $\mathrm{Mp}: 176-178{ }^{\circ} \mathrm{C}$;

${ }^{1} \mathrm{H}$ NMR (400 MHz, DMSO- $\left.d_{6}\right)(\delta, \mathrm{ppm}): 7.93-7.88(\mathrm{~m}, 3 \mathrm{H}$, ArH), 7.81-7.79 (m, 3H, ArH), 7.73-7.63 (m, 4H, ArH), 7.51$7.35(\mathrm{~m}, 7 \mathrm{H}, \mathrm{ArH}), 2.97\left(\mathrm{~s}, 2 \mathrm{H}, \mathrm{CH}_{2}\right), 1.82-1.81\left(\mathrm{~m}, 2 \mathrm{H}, \mathrm{CH}_{2}\right)$, $1.09\left(\mathrm{~s}, 3 \mathrm{H}, \mathrm{CH}_{3}\right)$;

${ }^{13} \mathrm{C}$ NMR (100 MHz, DMSO- $\left.d_{6}\right)(\delta, \mathrm{ppm}): 172.4,144.8,143.9$, $143.7,140.4,136.2,136.1,133.3,132.2,132.1,130.9,129.5$, $129.4,128.9,128.7,128.2,128.1,128.1,127.8,127.3,126.0$, 121.8, 121.7, 118.5, 116.6, 114.5, 107.4, 36.2, 18.4, 14.0;

IR $\left(\mathrm{KBr}, v, \mathrm{~cm}^{-1}\right): 3055,1763,1645,1619,1586,1481,1470$, 1451, 1416, 1358;

HRMS (ESI): $\mathrm{m} / \mathrm{z}$ calcd for: $\mathrm{C}_{34} \mathrm{H}_{24} \mathrm{ClNNaO}_{2}, \quad 536.1393$ $[\mathrm{M}+\mathrm{Na}]^{+}$; found: 536.1408 .

\section{7-(4-Bromophenyl)-9-methyl-7H-acenaphtho[1,2-b]indol-11-} yl isobutyrate (8r)

A red-orange solid: $0.347 \mathrm{~g}$, yield $70 \%$; Mp: $159-161{ }^{\circ} \mathrm{C}$;

${ }^{1} \mathrm{H}$ NMR (400 MHz, DMSO- $\left.d_{6}\right)(\delta, \mathrm{ppm}): 7.93$ (d, 2H, $J=6.8$ $\mathrm{Hz}, \mathrm{ArH}), 7.86(\mathrm{~d}, 1 \mathrm{H}, J=7.2 \mathrm{~Hz}, \mathrm{ArH}), 7.79-7.74(\mathrm{~m}, 3 \mathrm{H}$, ArH), 7.64-7.61 (m, 2H, ArH), 7.50 (s, 1H, ArH), 7.39-7.38 (m, 1H, ArH), 7.12 (s, 1H, ArH), 6.88 (s, 1H, ArH), 3.27-3.24 (m, $1 \mathrm{H}, \mathrm{CH}), 2.41\left(\mathrm{~s}, 3 \mathrm{H}, \mathrm{CH}_{3}\right), 1.40\left(\mathrm{~d}, 6 \mathrm{H}, J=5.2 \mathrm{~Hz}, \mathrm{CH}_{3}\right)$;

${ }^{13} \mathrm{C}$ NMR (100 MHz, DMSO- $\left.d_{6}\right)(\delta, \mathrm{ppm}): 175.9,143.7,143.6$, $143.3,136.8,133.7,133.6,132.4,131.9,129.5,128.8,128.5$, $128.3,128.1,125.9,121.6,121.4,118.6,116.4,115.4,109.4$, 100.0, 34.2, 31.1, 21.7, 19.5;

IR $\left(\mathrm{KBr}, v, \mathrm{~cm}^{-1}\right): 3036,1757,1623,1607,1582,1514,1482$, 1444, 1414, 1345;

HRMS (ESI): $\mathrm{m} / \mathrm{z}$ calcd for: $\mathrm{C}_{29} \mathrm{H}_{22} \mathrm{BrNNaO}_{2}, \quad 518.0732$ [M+Na $]^{+}$; found: 518.0756 .

\section{7-(4-Chlorophenyl)-9-phenyl-7H-acenaphtho[1,2-b]indol-11-} yl isobutyrate (8s)

A red-orange solid: $0.328 \mathrm{~g}$, yield $64 \%$; Mp: $175-177{ }^{\circ} \mathrm{C}$;

${ }^{1} \mathrm{H}$ NMR $\left(400 \mathrm{MHz}, \mathrm{DMSO}-d_{6}\right)(\delta, \mathrm{ppm}): 7.96-7.90(\mathrm{~m}, 3 \mathrm{H}$, ArH), 7.80 (t, 3H, $J=7.6 \mathrm{~Hz}, \mathrm{ArH}), 7.71-7.64(\mathrm{~m}, 4 \mathrm{H}, \mathrm{ArH})$, 7.55-7.38 (m, 7H, ArH), 3.31-3.26 (m, 1H, CH), $1.44(\mathrm{~d}, 6 \mathrm{H}, J=$ $\left.6.4 \mathrm{~Hz}, \mathrm{CH}_{3}\right)$;

${ }^{13} \mathrm{C}$ NMR (100 MHz, DMSO- $\left.d_{6}\right)(\delta, \mathrm{ppm}): 175.9,144.9,144.0$, $143.9,140.4,136.2,136.1,133.4,132.2,132.1,130.9,129.6$, $129.4,128.8(4), 128.7(8), 128.3,128.1(3), 128.0(9), 127.8$, 127.4, 126.0, 121.9, 121.6, 118.4, 116.7, 114.3, 107.4, 34.3, 19.5; IR $\left(\mathrm{KBr}, v, \mathrm{~cm}^{-1}\right): 3051,1732,1678,1579,1546,1503,1494$, 1473, 1389, 1354;

HRMS (ESI): $\mathrm{m} / \mathrm{z}$ calcd for: $\mathrm{C}_{34} \mathrm{H}_{24} \mathrm{ClNNaO}_{2}, \quad 536.1393$ $[\mathrm{M}+\mathrm{Na}]^{+}$; found: 536.1411 .

Supporting Information (see footnote on the first page of this article): .... ((Please delete if not appropriate.))
We are grateful for financial support from the NSFC (No. 21332005), PAPD of Jiangsu Higher Education Institutions, Robert A. Welch Foundation (D-1361, USA) and NIH (R33DA031860, USA), the Outstanding Youth Fund of JSNU (YQ2015003), NSF of Jiangsu Province (BK20151163), and the Open Foundation of Jiangsu Key Laboratory (K201505).

\section{References and notes}

(1) (a) Melhado, A. D.; Brenzovitch, W. E.; Lackner, A. D.; Toste, F. D. J. Am. Chem. Soc. 2010, 132, 8885-8887; (b) Adams, G. L.; Carroll, P. J.; Smith, A. B. III J. Am. Chem. Soc. 2013, 135, 519-528; (c) Snyder, S. A.; Breazzano, S. P.; Ross, A. G.; Lin, Y.; Zografos, A. L. J. Am. Chem. Soc. 2009, 131, 1753-1765; (d) Jiang, B.; Feng, B.-M.; Wang, S.-L.; Tu, S.-J.; Li, G. Chem. Eur. J. 2012, 18, 9823-9826; (e) Qiu, J.K.; Jiang, B.; Zhu, Y.-L.; Hao, W.-J.; Wang, D.-C.; Sun, J.; Wei, P.; Tu, S.-J.; Li, G. J. Am. Chem. Soc. 2015, 137, 8928-8931.

(2) (a) Yoder, R. A.; Johnston, J. N. Chem. Rev. 2005, 105, 4730-4756; (b) Sutherland, J. K. In Comprehensive Organic Synthesis; Trost, B. M., Ed.; Pergamon Press: Elmsford, NY, 1991; Vol. 1, p 341. (c) Chen, Z.-Z.; Liu, S.; Hao, W.-J.; Xu, G.; Wu, S.; Miao, J.-N.; Jiang, B.; Wang, S.-L.; Tu, S.-J.; Li, G. Chem. Sci. 2015, 6, 6654-6658; (d) Gao, Q.; Zhou, P.; Liu, F.; Hao, W.-J.; Yao, C.; Jiang, B.; Tu, S.-J. Chem. Commun. 2015, 51, 9519-9522. (f) Zhu, Y.-L.; Jiang, B.; Hao, W.-J.; Qiu, J.-K.; Sun, J.; Wang, D.-C.; Wei, P.; Wang, A.-F.; Li, G.; Tu, S.-J. Org. Lett. 2015, 17, 6078-6081.

(3) (a) Yamada, F.; Makita, Y.; Suzuki, T.; Somei, M. Chem. Pharm. Bull. 1985, 33, 2162-2163; (b) Hegedus, L. S.; Toro, J. L.; Miles, W. H.; Harrington, P. J. J. Org. Chem. 1987, 52, 3319-3322; (c) Yamada, K.; Namerikawa, Y.; Haruyama, T.; Miwa, Y.; Yanada, R.; Ishikura, M.; Eur. J. Org. Chem. 2009, 5752-5759; (d) Grougnet, R.; Magiatis, P.; Fokialakis, N.; Mitaku, S.; Skaltsounis, A.-L.; Tillequin, F.; Sevenet, T.; Litaudon, M. J. Nat. Prod. 2005, 68, 1083-1086; (e) Tillequin, F.; Koch, M.; Bert, M.; Sevenet, T. J. Nat. Prod. 1979, 42, 92-95.

(4) (a) Choi, T. A.; Czerwonka, R.; Frohner, W.; Krahl, M. P.; Reddy, K. R.; Franzbau, S. G.; Knolker, H.-J. ChemMedChem 2006, 1, 812-815; (b) Lescot, E.; Muzard, G.; Markovits, J.; Belleney, J.; Roques, B. P.; Le Pecq, J.-B. J. Med. Chem. 1986, 29, 1731-1737; (c) Thevissen, K.; Marchand, A.; Chaltin, P.; Meert, E. M. K.; Cammue, B. P. A. Curr. Med. Chem. 2009, 16, 2205-2211; (d) Conchon, E.; Anizon, F.; Aboab, B.; Prudhomme, M. J. Med. Chem. 2007, 50, 4669-4680; (e) Alper, P. B.; Marsilje, T. H.; Mutnick, D.; Lu, W.; Chatterjee, A.; Roberts, M. J.; He, Y.; Karanewsky, D. S.; Chow, D.; Lao, J.; Gerken, A.; Tuntland, T.; Liu, B.; Chang, J.; Gordon, P.; Seidel, H. M.; Tian, S.-S. Bioorg.Med. Chem. Lett. 2008, 18, 5255-5258.

(5) (a) Penning, T. M. J. Pharm. Sci. 1985, 74, 651-654; (b) Kleinwächter, P.; Schlegel, B.; Groth, I.; Härtl, A.; Gräfe, U. J. Antibiot. 2001, 54, 510-512.

(6) (a) Bal, C.; Baldeyrou, B.; Moz, F.; Lansiaux, A.; Colson, P.; KrausBerthier, L.; Leonce, S.; Pierre, A.; oussard, B. M.; Rousseau, A.; Wierzbicki, M.; Bailly, C. Biochem. Pharmacol. 2004, 68, 1911-1922; (b) Wierzbicki, M.; Boussard, M. F.; Rousseau, A.; Boutin, A.; Delagrange, P. EP 1245565, 2002.

(7) Brown, D. W.; Graupner, P. R.; Sainsbury, M.; Shertzer, H. G. Tetrahedron 1991, 47, 4383-4408.

(8) (a) Butera, J. A.; Antane, S. A.; Hirth, B.; Lennox, J. R.; Sheldon, J. H.; Norton, N. W.; Warga, D.; Argentieri, T. M. Bioorg. Med. Chem. Lett. 2001, 11, 2093-2097; (b) Antane, S. A.; Butera, J. A.; Lennox, J. R. US 2001047026, 2001

(9) (a) Kraus, A.; Wu, T. Tetrahedron Lett. 2006, 47, 7801-7803; (b) Scopton, A.; Kelly, T. R. J. Org. Chem. 2005, 70, 10004-10012; (c) Hemmerling, H. J.; Reiss, G. Synthesis 2009, 985-999; (d) Janreddy, D.; Kavala, V.; Bosco, J. W. J.; Kuo, C.-W.; Yao, C.-F. Eur. J. Org. Chem. 2011, 2360-2365; (e) Cacchi, S.; Fabrizi, G. Chem. Rev. 2005, 105, 2873-2920; (f) Humphrey, G. R.; Kuethe, J. T. Chem. Rev. 2006, 106, 2875-2911.

(10) (a) Campo, M. A.; Larock, R. C. J. Org. Chem. 2002, 67, 5616-5620; (b) Chernyak, N.; Tilly, D.; Li, Z.; Gevorgyan, V. Arkivoc 2011, 5, 7698; (c) Zhao, Z.; Jaworski, A.; Piel, I.; Snieckus, V. Org. Lett. 2008, 10, 2617-2620.

(11) (a) Nair, V.; Rajesh, C.; Vinod, A. U.; Bindu, S.; Sreekanth, A. R.; Mathen, J. S.; Balagopal, L. Acc. Chem. Res. 2003, 36, 899-907; (b) Ganem, B. Acc. Chem. Res. 2009, 42, 463-472; (c) Domling, A.; Wang, W.; Wang, K. Chem. Rev. 2012, 112, 3083-3135; (d) D'Souza, D. M.; Mueller, T. J. J. Chem. Soc. Rev. 2007, 36, 1095-1108; (e) Ruijter, E.; Scheffelaar, R.; Orru, R. V. A. Angew. Chem., Int. Ed. 2011, 50, 6234-6246; (f) Sunderhaus, J. D.; Martin, S. F. Chem.-Eur. J. 2009, 15, 1300-1308. 
(12) (a) Estevez, V.; Villacampa, M.; Menendez, J. C. Chem. Soc. Rev. 2010, 39, 4402-4421; (b) Shiri, M. Chem. Rev. 2012, 112, 3508-3549; (c) Jiang, B.; Rajale, T.; Wever, W.; Tu, S.-J.; Li, G. Chem.-Asian J. 2010, 5, 2318-2335; (d) Isambert, N.; Duque, M.; del, M. S.; Plaquevent, J.-C.; Genisson, Y.; Rodriguez, J.; Constantieux, T. Chem. Soc. Rev. 2011, 40, 1347-1357; (e) Tietze, L. F. Chem. Rev. 1996, 96, 115-136.

(13) (a) Tietze, L. F.; Brasche, G.; Gerike, K. Domino Reactions in Organic Chemistry; Wiley-VCH: Weinheim, Germany, 2006; (b) Zhu, J.-P.; Bienayme, H. Multicomponent Reactions; Wiley-VCH: Weinheim, Germany, 2004; (c) Toure, B. B.; Hall, D. G. Chem. Rev., 2009, 109, 4439-4486.

(14) (a) Jiang, B.; Yi, M.-S.; Shi, F.; Tu, S.-J.; Pindi, S.; McDowell, P.; Li, G. Chem. Commun. 2012, 48, 808-810; (b) Jiang, B.; Li, Q.-Y.; Zhang, H.; Tu, S.-J.; Pindi, S.; Li, G. Org. Lett. 2012, 14, 700-703; (c) Jiang, B.; Yi, M.-S.; Tu, M.-S.; Wang, S.-L.; Tu, S.-J. Adv. Synth. Catal. 2012, 354, 2504-2510; (d) Jiang, B.; Li, Y.; Tu, M.-S.; Wang, S.-L.; Tu, S.-J.; Li, G. J. Org. Chem. 2012, 77, 7497-7505.

(15) Zhang, J.-J.; Feng, X.; Liu, X.-C.; Huang, Z.-B.; Shi, D.-Q. Mol. Divers. 2014, 18, 727-736

(16) Chen, X.-B.; Luo, T.-B.; Gou, G.-Z.; Wang, J.; Liu, W.; Lin, J. Asian J. Org. Chem. 2015, 4, 921-928

(17) (a) An, G.-H.; Seifert, C.; Li, G. Org. Biomol. Chem. 2015, 13, 16001617; (b) Kaur, P.; Pindi, S.; Wever, W.; Rajale, T.; Li, G. J. Org. Chem. 2010, 75, 5144-5150; (c) Cheng, C.; Jiang, B.; Tu, S.-J.; Li, G. Green Chem. 2011, 13, 2107-2115; (d) Kaur, P.; Wever, W.; Pindi, S.; Milles, R.; Gu, P.; Shi, M.; Li, G. Green Chem. 2011, 13, 1288-1292; (e) Kattamuri, P. V.; Ai, T.; Pindi, S.; Sun, Y.; Gu, P.; Shi, M.; Li, G. J. Org. Chem. 2011, 76, 2792-2797.

(18) Jiang, B.; Li, Q.-Y.; Tu, S.-J.; Li, G. Org. Lett. 2012, 14, 5210-5213.

(19) Crystal data for $\mathbf{3 j}: \mathrm{C}_{27} \mathrm{H}_{23} \mathrm{NO}_{3}, M r=409.46$, Monoclinic, $a=$ $13.7175(14) \AA, b=16.5978(16) \AA, c=18.6764(18) \AA, U=4250.7(7)$ $\AA^{3}, T=298(2) \mathrm{K}$, space group $\mathrm{P} 2(1) / \mathrm{c}, Z=8,7428$ reflections measured, 7428 unique $\left(\mathrm{R}_{\text {int }}=0.0000\right)$ which were used in all calculation. The final $w R\left(F_{2}\right)$ was0.3590 (all data)

(20) Crystal data for 4f: $\mathrm{C}_{29} \mathrm{H}_{23} \mathrm{Cl}_{2} \mathrm{NO}_{3}, M r=504.38$, Triclinic, $a=$ 10.3883(11) ̊, $b=10.7901(12) \AA, c=13.1361(15) \AA, U=1260.8(2)$ $\AA^{3}, T=298(2) \mathrm{K}$, space group P-1, $Z=2,6436$ reflections measured, 4370 unique $\left(\mathrm{R}_{\mathrm{int}}=0.0292\right)$ which were used in all calculation. The final $w R\left(F_{2}\right)$ was0.1150 (all data).

(21) Crystal data for $6 c$ : $\mathrm{C}_{26} \mathrm{H}_{18} \mathrm{BrNO}, M r=440.32$, Monoclinic, $a=$ 9.2942(8) ̊, $b=11.9911(11) \AA, c=17.9266(17) \AA, U=1996.0(3) \AA^{3}$ $T=293(2) \mathrm{K}$, space group $\mathrm{P} 2(1) / \mathrm{c}, Z=4,12878$ reflections measured, 3518 unique $\left(\mathrm{R}_{\text {int }}=0.1012\right)$ which were used in all calculation. The final $w R\left(F_{2}\right)$ was0.1131 (all data).

(22) Crystal data for 8e: $\mathrm{C}_{32} \mathrm{H}_{20} \mathrm{ClNO}_{2}, M r=485.94$, Monoclinic, $a=$ 12.5369(9) $\AA, b=25.344(2) \AA, c=7.6693(6) \AA, U=2334.2(3) \AA^{3}, T$ $=298(2) \mathrm{K}$, space group $\mathrm{P} 2(1) / \mathrm{c}, Z=4,11671$ reflections measured, 4093 unique $\left(\mathrm{R}_{\text {int }}=0.0825\right)$ which were used in all calculation. The final $w R\left(F_{2}\right)$ was0.1252 (all data). 


\section{Graphical Abstract}

To create your abstract, type over the instructions in the template box below.

Fonts or abstract dimensions should not be changed

Domino reactions of cyclic enaminones

Leave this area blank for abstract info.

leading to selective synthesis of pentacyclic indoles and its functionalization

Wei Fan, Yan-Rong Li, Qun Li, Bo Jiang, Guigen Li
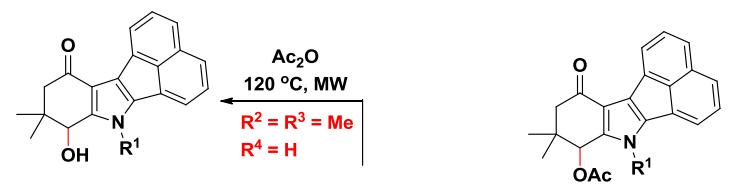

$\mathrm{Ac}_{2} \mathrm{O}$

$120^{\circ} \mathrm{C}, \mathrm{MW}$

$R^{2}=R^{3}=H$

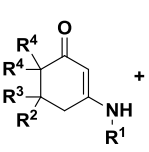
OAC $R^{1}$

$\mathrm{R}^{4}=\mathrm{Me} \quad \mathrm{R}^{2} \quad \underset{\mathbf{R}^{1}}{\mathrm{NH}}$
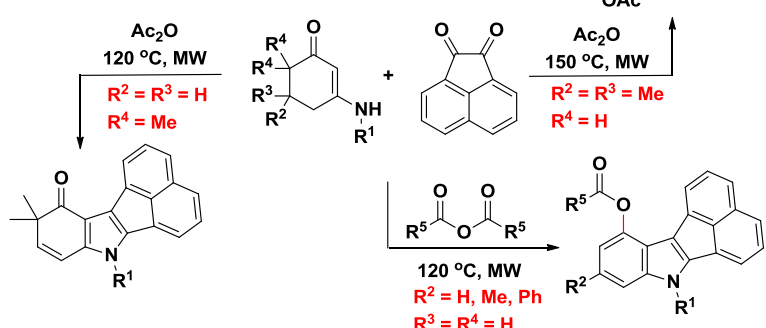